\title{
Weyl calculus in QED I. The unitary group.
}

\author{
L. Amour ${ }^{1}$, R. Lascar ${ }^{2}$ and J. Nourrigat ${ }^{1}$ \\ ${ }^{1}$ Université of Reims $\quad{ }^{2}$ Université Paris 7, Denis Diderot
}

\begin{abstract}
In this work, we consider fixed $1 / 2$ spin particles interacting with the quantized radiation field in the context of quantum electrodynamics (QED). We investigate the time evolution operator in studying the reduced propagator (interaction picture). We first prove that this propagator belongs to the class of infinite dimensional Weyl pseudodifferential operators recently introduced in 3 . on Wiener spaces. We give a semiclassical expansion of the symbol of the reduced propagator up to any order with estimates on the remainder terms. Next, taking into account analyticity properties for the Weyl symbol of the reduced propagator, we derive estimates concerning transition probabilities between coherent states.
\end{abstract}

Keywords: Semiclassical analysis, pseudodifferential operators, infinite dimensional analysis, Weyl calculus, quantum electrodynamics, spin-boson model, propagators, reduced propagator, interaction picture, transition probability.

MSC 2010: 35S05, 81V10.

\section{Contents}

1 Introduction.

2 Usual Hilbert spaces in QED. 6

2.1 The one photon Hilbert space. . . . . . . . . . . . . . . . 6

2.2 The quantized field Hilbert space. . . . . . . . . . . . . . . . . . . 7

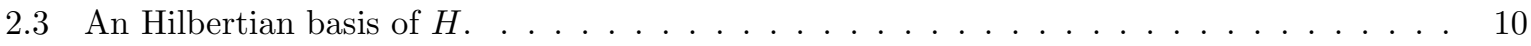

3 Usual operators in QED. 11

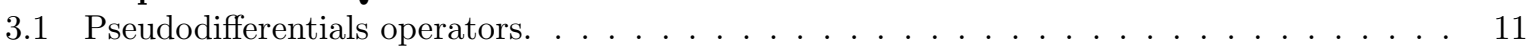

3.2 The functor $\Gamma$ and $\mathrm{d} \Gamma$. Photons number and energy. . . . . . . . . . . . . . 13

3.3 Field operators. . . . . . . . . . . . . . . . . . . . . 19

4 Spin-photon interaction model.

5 Proof of Theorem $1.1(i)(i i)(i v)$.

6 Proof of Theorem 1.1 (iii) (Semiclassical expansion of the reduced propagator symbol). 


\section{Introduction.}

The aim of this article consists in studying a model of one or several fixed $1 / 2$ spin particles in a constant magnetic field $\beta$ in the framework of quantum electrodynamics. This quantum model may be found in Reuse [35. It is related to the spin-boson model, for which spectral properties and some propagation results are known (see [24 [6] [1] 25] [14). A classical version of this model is introduced in [8] in 1946. The main goal of this paper is to describe the operator $e^{-i \frac{t}{h} H(h)}$ where $H(h)$ is the Hamiltonian of the model, using the infinite dimensional Weyl calculus recently developed in $[3$ and $[4$. Potential benefits of this calculus is to perform analysis when the semiclassical parameter $h$ tends to 0 .

The Hilbert space describing the quantized field without interaction is usually defined as the symmetrized Fock space $\mathcal{F}_{s}\left(H_{\mathbb{C}}\right)$ over the complexified of a real Hilbert space $H$ (completion of the direct sum of symmetric tensor products, see [33]). The space $H_{\mathbb{C}}$ describes the states of the field with exactly one photon. The definition of this space is not chosen here as the usual one but rather inspired by the space given by Lieb-Loss [30]. The definition is given in Section 2.1] The Hilbert space of the system of $N$ spins $(1 / 2)$ interacting with the quantized field is $\mathcal{F}_{s}\left(H_{\mathbb{C}}\right) \otimes\left(\mathbb{C}^{2}\right)^{\otimes N}$. The Hamiltonian is recalled in Section 4. Note that this operator is clearly simpler than the Pauli-Fierz operator where the particle is not fixed. See, e.g., Cohen-Tannoudji, Dupont-Roc, Grynberg [10] 11], Fröhlich [17] 18], Bach, Fröhlich, Segal [7. and Spohn [38].

Our goal in this paper and in forthcoming works is to adapt to the operator $H(h)$ defined below in Section 4, semiclassical results as those obtained in finite dimension, see e.g. 41. In order to do this, the Fock space $\mathcal{F}_{s}\left(H_{\mathbb{C}}\right)$ is less convenient than an $L^{2}$ space to which it is isomorphic. The definition of this $L^{2}$ space is reminded in Section 2.2. This space is related to an abstract Wiener space $(i, H, B)$ where $H$ is the real Hilbert space, $B$ is a suitable Banach space containing $H$ and $i$ the injection of $H$ into $B$. The precise assumptions fulfilled by $B$ are recalled in Section 2.2. For every $h>0$, the space $B$ is endowed with a gaussian measure with variance $h$ denoted by $\mu_{B, h}$. The main properties are recalled in [3]. The space $\mathcal{F}_{s}\left(H_{\mathbb{C}}\right)$ is then isomorphic to $L^{2}\left(B, \mu_{B, h / 2}\right)$ (see [37] [27])) also denoted here $\mathcal{H}_{p h}$. The choice of $h / 2$ as the variance is taken in order to agree with usual formulas of pseudodifferential calculus. Taking account of this isomorphism, the Hilbert space of the system considered here becomes $L^{2}\left(B, \mu_{B, h / 2}\right) \otimes\left(\mathbb{C}^{2}\right)^{\otimes N}=\mathcal{H}_{p h} \otimes\left(\mathbb{C}^{2}\right)^{\otimes N}$. The two spaces $H$ and $H_{\mathbb{C}}$ are specified in Section 2.1] The space $H_{\mathbb{C}}($ or $H \times H)$ is the single photon space. 
Two types of operators act in this space. The Hamiltonian $H_{p h}$ of the (non interacting) free field is an unbounded selfadjoint operator in $\mathcal{F}_{s}\left(H_{\mathbb{C}}\right)$ or in $L^{2}\left(B, \mu_{B, h / 2}\right)$, not described with the infinite dimensional Weyl calculus. It is usually defined as $H_{p h}=\mathrm{d} \Gamma\left(h M_{\omega}\right)$ where the functor $\Gamma$ and $\mathrm{d} \Gamma$ are standard operators defined in Fock spaces (see [34]) and $M_{\omega}$ is acting in the one photon space $H_{\mathbb{C}}$ (see Section [3.2). The notation $H_{0}=H_{p h} \otimes I$ is also used here. The operator $e^{i \frac{t}{h} H_{p h}}$ equals $\Gamma\left(e^{i t M_{\omega}}\right)$. This operator may be also viewed as a kind of metaplectic transform associated with the symplectic linear transform $\chi_{t}$ in $H^{2}$, which is also unitary (see Section 3.2 and see [29] for a metaplectic group in this context).

The other operators involved here are pseudodifferential operators. Weyl calculus associates bounded and unbounded operators in $\mathcal{H}_{p h}=L^{2}\left(B, \mu_{B, h / 2}\right)$ to suitable functions (symbols) defined on $H^{2}$. The definition of the operator associated with a symbol $F$ being a continuous linear form on $H^{2}$ is standard: it is usually called Segal field and defined with creation and annihilation operators. The operators $B_{j}(x)$ and $E_{j}(x)(1 \leq j \leq 3)$ associated with the components of the magnetic and electric fields at the point $x$ are defined in this way, for all $x$ in $\mathbb{R}^{3}$. See [17] [18] 38] and [34.

The Weyl calculus developed in [3] or [4] is an extension of the notion of Segal field. It enables to associate an operator (bounded or not) $O P_{h}^{\text {weyl }}(F)$ with some functions $F$ on $H^{2}$, for each $h>0$. To define a bounded operator, it is sufficient that the function $F$ belongs to a class $S_{2}(\mathcal{B}, \varepsilon)$ introduced in Definition 3.1 below, associated with an Hilbertian basis $\mathcal{B}=\left(e_{j}\right)$ of $H$ and with a summable sequence $\varepsilon=\left(\varepsilon_{j}\right)$. If the symbol $F$ takes values in $\mathcal{L}\left(\left(\mathbb{C}^{2}\right)^{\otimes N}\right)$ then the corresponding operator acts in $\mathcal{H}_{p h} \otimes\left(\mathbb{C}^{2}\right)^{\otimes N}$. Theorems 1.1 and 1.2 below essentially rely on Beals characterization Theorem (Theorem 1.2 of [4]).

The operator $H(h)$ denotes the Hamiltonian of the entire system, for all $h>0$ and $H_{0}=H_{p h} \otimes I$. These two operators are selfadjoint and share the same domain. The operator $H(h)$ is precisely defined in Section 4 .

The first main result is the following one.

Theorem 1.1. Let $H(h)$ be the Hamiltonian defined in Section 4 and $H_{0}=H_{p h} \otimes I$. Then, there exists a function $U(t, q, p, h)$ defined on $\mathbb{R} \times H^{2}$ with parameter $h>0$ and taking matrices values $\left(\right.$ in $\left.\mathcal{L}\left(\left(\mathbb{C}^{2}\right)^{\otimes N}\right)\right)$ fulfilling,

$$
e^{-i \frac{t}{h} H(h)}=e^{-i \frac{t}{h} H_{0}} U_{h}^{r e d}(t), \quad U_{h}^{r e d}(t)=O p_{h}^{\text {weyl }}(U(t, \cdot, h))
$$

and having the following properties.

(i) As a function of $(q, p)$, it belongs to the space $S_{\infty}^{\text {mat }}(\mathcal{B},|t| \varepsilon(t))$ introduced in Definition [3.1 associated with the Hilbertian basis $\mathcal{B}$ of Section 2.3 and the sequence $\varepsilon(t)$ of Proposition 5.1. 
(ii) Additionaly, this function is bounded in this space (in the sense of Definition 3.1) as $h$ runs over $(0,1)$ and $t$ belongs to a compact set of $\mathbb{R}$.

(iii) Moreover, the function $U(t, q, p, h)$ has a asymptotic expansion in powers of $h$ up to any order $m$,

$$
U(t, q, p, h)=\sum_{j=0}^{m} g_{j}(t, q, p) h^{j}+h^{m+1} R_{m}(t, q, p, h)
$$

where the $g_{j}(t, \cdot)$ and $R_{m}(t, \cdot, h)$ belong to $S_{\infty}^{m a t}(\mathcal{B}, K|t| \varepsilon(t))$, for some non negative real constant $K$. These functions are solutions to the differential system (89) (90).

(iv) In addition, the function $(q, p) \mapsto U(t, q, p, h)$ is analytic on $H^{2}$ and and there exits an holomorphic extension on $H_{\mathbb{C}}^{2}$ taking values in $\mathcal{L}\left(\left(\mathbb{C}^{2}\right)^{\otimes N}\right)$ and denoted $\mathcal{U}(t, q, p, h)$ which satisfies,

$$
|\mathcal{U}(t, q, p, h)| \leq M(h, t) e^{K|t||\operatorname{Im}(q, p)|}, \quad(q, p) \in H_{\mathbb{C}}^{2}
$$

where $M(t, h)>0$ is bounded when $h$ belongs to $(0,1)$ and $t$ to a compact in $\mathbb{R}$, and $K$ is some non negative real constant.

The proof uses a Calderón-Vaillancourt type result proved in [3] and a Beals characterization type theorem obtained in [4].

Note that the parameter $h$ appearing in the Hamiltonian $H(h)$ is also the parameter related to the variance of the Gaussian measure $\mu_{B, h / 2}$ defined above and associated to the $L^{2}$ space.

In the case of a single spin $(N=1)$, the system (89) satisfied by $g_{0}$ is related to Larmor precession. The expansion (2) is different from perturbative series (see, e.g., Section 8.3 of [19], formula (4.175) in [35], Section 4.2 in [32], Section 3.5 of $40, \ldots$ ), expansions in powers of the coupling constant. Indeed, the expansion (2) is a semiclassical expansion whereas perturbative series are not.

In finite dimension, the semiclassical Weyl calculus enables for instance to control the remainder terms up to any order of semiclassical expansions of various quantities or functions related to the Hamiltonian under consideration (for example, see [12, 15, 23, 31, 36, 41]). In (2), the precise control of the remainder terms relies on the estimates on the derivatives of $U(t, q, p, h)$ which are in Definition 3.1 of $S_{\infty}^{\text {mat }}(\mathcal{B},|t| \varepsilon(t)$ ) (See Section 6). Recently, the definition and some properties of the Weyl calculus are extended to the Wiener spaces setting: norm estimates ([3]), Beals type characterization ([4]). These properties are involved in the present work. One may think that others properties will be soon also extended to the Wiener spaces setting and the resulting applications may concerned the semiclasical expansion and control of the 
remainder terms of the time evolution for some average values of some physical observables (Ehrenfest type result). This study should probably be local in time, as in finite dimension. Another tool for semiclassical analysis comes from coherent states depending on the parameter $h$ (see definition in Section 3.1) and their semiclassical evolution for the Hamiltonian $H(h)$ defined in Section 4 is given in [5]. Here, the applications are only the expansion (2) and the result below (Theorem 1.2) which is a consequence of the analyticity properties of the symbol.

Theorem $1.1(i)(i i)(i v)$ is proved in Section 5 and the semiclassical expansion of the symbol $U(t, q, p, h)$ (function defined on $\mathbb{R} \times H^{2}$ and taking values in $\mathcal{L}\left(\left(\mathbb{C}^{2}\right)^{\otimes N}\right)$ ) of Theorem 1.1 (iii) is given in Section 6 ,

Operators with analytic symbols are introduced in 9 in finite dimension. An application of the analyticity properties is to derive estimates of transition probabilities between coherent states. Coherent states $\Psi_{X, h}$ are elements of the Fock space, or alternatively of the $L^{2}$ space which is isomorphic, indexed by an element $X=(a, b)$ of $H^{2}$ where $H$ is the one photon real Hilbert space. Moreover, the coherent states depend on the semiclassical parameter $h>0$ and their definitions are reminded in (20) (21). In the case of finite dimension, if a propagator is known to be the composition of a pseudodifferential operator with a metaplectic operator as in (1) then an estimate of the transition probability between coherent states is given in [39. The analyticity of the symbol here improves this estimate. Let us recall that the symplectic map $\chi_{t}$ in $H^{2}$ defined in (34) reflects the free dynamics of photons between times 0 and $t$.

Theorem 1.2 below is proved in Section 7 .

Theorem 1.2. For all $X$ and $Y$ in $H^{2}$, for any $a$ and $b$ in $\left(\mathbb{C}^{2}\right)^{\otimes N}$ with norm 1 , the following estimate holds,

$$
\left|<e^{i \frac{t}{h} H(h)}\left(\Psi_{X h} \otimes a\right), \Psi_{Y h} \otimes b>\right| \leq M(h, t) e^{K|t|\left|X-\chi_{t}^{-1}(Y)\right|-\frac{1}{4 h}\left|X-\chi_{t}^{-1}(Y)\right|^{2}}
$$

where $\chi_{t}$ is the symplectic map in $H^{2}$ defined in 34) and where $M(h, t)$ and $K$ are the constants of Theorem 1.1 (iv).

The benefit of Theorem 1.2 comes from the comparison with standard results. It is proved in Unterberger [39] that, if $A_{h}$ is an operator associated by the Weyl calculus in finite dimension with a function which is bounded together with all of its derivatives and if the $\Psi_{X h}\left(X \in \mathbb{R}^{2 n}\right)$ are the usual coherent states then, for all integers $N$, there exists $C_{N}>0$ such that, for all $X$ and $Y$ in $\mathbb{R}^{2 n}$, for any $h$ in $(0,1)$,

$$
\left|<A \Psi_{X h}, \Psi_{Y h}>\right| \leq C_{N}\left[1+\frac{|X-Y|}{\sqrt{h}}\right]^{-N} .
$$

Note the two differences with Theorem 1.2 Namely, the free photons evolution leads to replace $Y$ by 
$\chi_{t}(Y)$ and the analytic properties of the symbol enables to improve the rapid decay estimate which is now replaced by an exponential decay estimate.

A model for a fixed $1 / 2$ spin particle in a constant magnetic field interacting with the quantized field and also interacting with a rotating (non quantized) magnetic field may also be considered. It is seen in Section 4 that such a model may be reduced with a suitable transform to the case where only the constant field is not quantized. Thus, a standard method in quantum mechanics remains valid in QED (see Theorem 4.2).

Notations. The notation ( , ) stands here for real scalar products on $H$ or $H^{2}$ and $<,>$ denotes Hermitian products, antilinear with respect to the second variables. These products are used on $\mathcal{H}_{p h}$, $\left(\mathbb{C}^{2}\right)^{\otimes N}$, and on $H^{2}$ when identified to the complexified $H_{\mathbb{C}}$. In that case, it is written as,

$$
<X, Y>=(X, Y)+i \sigma(X, Y), \quad X, Y \in H_{\mathbb{C}}
$$

\section{Usual Hilbert spaces in QED.}

\subsection{The one photon Hilbert space.}

The space $H$ (resp. $H_{\mathbb{C}}$ ) stands for the set of mappings $f=\left(f_{1}, f_{2}, f_{3}\right)$ with $f_{j}$ belonging to $L^{2}\left(\mathbb{R}^{3}\right)$ and taking real (resp. complex) values, satisfying

$$
k_{1} f_{1}(k)+k_{2} f_{2}(k)+k_{3} f_{3}(k)=0 .
$$

This space is endowed with the norm

$$
|f|^{2}=\sum_{j=1}^{3} \int_{\mathbb{R}^{3}}\left|f_{j}(k)\right|^{2} d k .
$$

The space $H_{\mathbb{C}}$ is here the Hilbert space corresponding to a unique photon. It is the space of divergence free for Fourier transforms of vector fields. As later seen, it is also related to the set of all possible initial data for Maxwell equations in vacuum (without interaction).

Let us also mention a more common way to introduce this space. For all $k \in \mathbb{R}^{3}$ such that $k_{1}^{2}+k_{2}^{2} \neq 0$, one can find $e_{1}(k)$ and $e_{2}(k)$ in $k^{\perp}$, being an orthonormal basis of $k^{\perp}$ and $C^{\infty}$ regular with respect to $k$ in its domain of definition. For each $f$ in $H$ and for any $k$ in its domain of definition, set $f_{\lambda}(k)=f(k) \cdot e_{\lambda}(k)$ $(\lambda=1,2)$. One has,

$$
f(k)=f_{1}(k) e_{1}(k)+f_{2}(k) e_{2}(k)
$$


and

$$
|f|^{2}=\sum_{\lambda=1,2} \int_{R^{3}}\left|f_{\lambda}(k)\right|^{2} d k
$$

for any $k$ in the domain. Thus, $H$ is isomorphic to $L^{2}\left(\mathbb{R}^{3}\right)^{2}$ or to $L^{2}\left(\mathbb{R}^{3} \times\{1,2\}\right)$. This is the usual way but less convenient, since a singularity now appears in the set of $k \in \mathbb{R}^{3}$ with $k_{1}^{2}+k_{2}^{2}=0$.

\subsection{The quantized field Hilbert space.}

It is the symmetrized Fock space $\mathcal{F}_{s}\left(H_{\mathbb{C}}\right)$ over $H_{\mathbb{C}}$. For pseudodifferential analysis, we rather use an $L^{2}$ space, to which it is isomorphic. To this end, a Banach space $B$ containing $H$ may be found, together with a suitable measure. Let us first recall the hypotheses fulfilled by $B$. For all finite dimension subspaces $E$ in $H$ and for all $h>0$, a measure $\mu_{E, h}$ is defined by setting,

$$
\mu_{E, h}(\Omega)=(2 \pi h)^{-\operatorname{dim}(E) / 2} \int_{\Omega} e^{-\frac{|y|^{2}}{2 h}} d y,
$$

for all Borel sets $\Omega$ in $E$.

Definition 2.1. ([21][22][28]) Let $H$ be real separable Hilbert space with the norm $|\cdot|$. Define on $H$ another norm $N$ satisfying for some constant $C>0$,

$$
N(x) \leq C|x|
$$

The norm $N$ is called measurable if for all $\varepsilon>0$ and for all $h>0$, there exists a finite dimensional subspace $F$ in $H$ such that, for any finite dimensional subspaces $E$ orthogonal to $F$,

$$
\mu_{E, h}(\{x \in E, N(x)>\varepsilon\})<\varepsilon
$$

where $\mu_{E, h}$ is defined in (8).

Such a norm can always be found. The completion $B$ of $H$ for such a norm satisfies,

$$
B^{\prime} \subset H \subset B
$$

each space being dense in the next one. Denoting $i$ the injection from $H$ into $B,(i, H, B)$ is called a Wiener space. Then, for all $h>0$, the Borel $\sigma$-algebra on $B$ is equipped with a measure denoted by $\mu_{B, h}$, related through natural formulas to the measures $\mu_{E, h}$ defined in (8), for all finite dimensional subspaces $E$ of $H$. Then, the Fock space $\mathcal{F}_{s}\left(H_{\mathbb{C}}\right)$ becomes isomorphic to $L^{2}\left(B, \mu_{B, h}\right)$, for all $h$. To fit 
with standard formulas of pseudodifferential calculus, we choose $L^{2}\left(B, \mu_{B, h / 2}\right)$ as the Hilbert space of the quantized field, isomorphic to the Fock space.

We now specify the choice of space $B$ suitable for the space $H$ defined in Section 2.1 It is already known that, if $D$ is an injective Hilbert-Schmidt operator in a Hilbert space $H$, then the norm $x \rightarrow|D x|$ is measurable on $H\left([22]\right.$ ), example 2, p. 92). In our case, with $H^{0}$ being the space $L^{2}\left(\mathbb{R}^{3}\right)^{3}$, the operator

$$
D=\left(I-\Delta_{k}+|k|^{2}\right)^{-m}, \quad m>\frac{3}{2}
$$

acting on the three components, is Hilbert Schmidt in $H^{0}$, and the norm $f \mapsto N(f)=|D f|$ is therefore measurable on $H^{0}$. It is easily seen that a measurable norm restricted to a closed subset is a measurable norm on that subset. The subset $H$ of $H^{0}$ is closed. Consequently, one may choose as a space $B$, the completion of $H$ defined above for the norm $N$. Thus $(i, H, B)$ is a Wiener space.

Usually in field theory (see [37]), the space $\mathcal{S}^{\prime}\left(\mathbb{R}^{3}\right)$ is equipped with a probability measure and the space $L^{2}\left(\mathcal{S}^{\prime}\left(\mathbb{R}^{3}\right)\right)$ is isomorphic to $\mathcal{F}_{s}\left(H_{\mathbb{C}}\right)$. In order to use the results in [3] and 4], which themselves use results of Gross, Kuo and Janson (20] 21] 22] 27] 28]), the space $\mathcal{S}^{\prime}\left(\mathbb{R}^{3}\right)$ has to be replaced with a suitable Banach space $B$ containing the space $H$ of Section 2.1 It is on the Borel $\sigma$-algebra of this space $B$ (which is not unique) that is defined the probability measure that is used here. If instead of the space defined in Section 2.1. we simply had $L^{2}\left(\mathbb{R}^{3}\right)$, then the space $B$ would be a negative order Sobolev space sufficiently large, actually relatively close to $\mathcal{S}^{\prime}\left(\mathbb{R}^{3}\right)$.

For all $a$ in $H$, one uses a function $\ell_{a}$ defined on $B$ in the following way. When $a$ belongs to $B^{\prime} \subset H$, one has $\ell_{a}(x)=a(x)$. If $a$ is $H$, it is approximated by a sequence $\left(a_{j}\right)$ in $B^{\prime}$. The sequence $\ell_{a_{j}}$ is proved to be a Cauchy sequence in $L^{2}\left(B, \mu_{B, h / 2}\right)$, and we denote by $\ell_{a}$ its limit.

The following subspace $\mathcal{D}$ of $L^{2}\left(B, \mu_{B, h / 2}\right)$ is often used in the sequel.

Definition 2.2. For all finite dimensional subspaces $E$ of $H, \mathcal{D}_{E}$ denotes the space of functions $f: B \rightarrow \mathbb{C}$ satisfying,

i) The function $f$ is written as $g \circ P_{E}$ where $g$ is a continuous function from $E$ to $\mathbb{C}$ and where $P_{E}$ is the mapping from $B$ to $E$ defined as following, when choosing an orthonormal basis $\left\{u_{1}, \ldots, u_{n}\right\}$ of $E$,

$$
P_{E}(x)=\sum_{j=1}^{n} \ell_{u_{j}}(x) u_{j}, \quad \text { for a.e. } x \in B
$$

(This mapping $P_{E}$ is independent on the chosen basis).

ii) The function $E^{2} \ni X \mapsto<f, \psi_{X h}>$ belongs to the Schwartz space $\mathcal{S}\left(E^{2}\right)$. 
The union of all subspaces $\mathcal{D}_{E}$ is denoted by $D$.

According to Definition 4.4 of [3] we say that a continuous function $f$ on $H$ has a stochastic extension denoted $\widetilde{f}$ in $L^{p}\left(B, \mu_{B, h}\right)(1 \leq p<\infty)$ if, for all non decreasing sequence of finite dimensional subspaces $\left(E_{n}\right)$ in $H$ with a dense union in $H$, the sequence $f \circ P_{E_{n}}$ is in $L^{p}\left(B, \mu_{B, h}\right)$ and converges to $\tilde{f}$ in this space. Examples are found in Section 8.2 of $[3]$.

The next property is used in the following.

Proposition 2.3. Let $f: H \rightarrow \mathbb{C}$ be a continuous function with a stochastic extension $\widetilde{f}$ in $L^{1}\left(B, \mu_{B, h}\right)$. Let $\chi: H \rightarrow H$ be a unitary continuous linear mapping. Then $g=f \circ \chi$ has a stochastic extension $\widetilde{g}$ in $L^{1}\left(B, \mu_{B, h}\right)$ and one has,

$$
\int_{B} \widetilde{g}(y) d \mu_{B, h}(y)=\int_{B} \widetilde{f}(x) d \mu_{B, h}(x)
$$

Proof. Let $\left(E_{n}\right)$ be a non decreasing sequence of finite dimensional subspaces in $H$ whose union in dense in $H$. Set $F_{n}=\chi\left(E_{n}\right)$. The sequence $\left(F_{n}\right)$ shares the same properties. The hypothesis on $f$ implies that the sequence $f \circ P_{F_{n}}$ is a Cauchy sequence in $L^{1}\left(B, \mu_{B, h}\right)$. For all finite dimensional subsets $X$ and $Y$ of $H$ with $X \subset Y$, let $\pi_{X Y}$ be the orthogonal projection from $Y$ to $X$. According to the transfer Theorem, the assumption gives when $m<n$, that the integral,

$$
I_{m n}=\int_{F_{n}}\left|f(x)-f\left(\pi_{F_{m}, F_{n}}(x)\right)\right| d \mu_{F_{n}, h}(x)
$$

goes to 0 as $m=\inf (m, n)$ tends to infinity. The change of variables $x=\chi(y)$ shows that,

$$
I_{m n}=\int_{E_{n}}\left|g(y)-g\left(\pi_{E_{m}, E_{n}}(y)\right)\right| d \mu_{E_{n}, h}(y) .
$$

Using the transfer Theorem, this proves that $g \circ P_{E_{n}}$ is a Cauchy sequence in $L^{1}\left(B, \mu_{B, h}\right)$. Since the sequence $E_{n}$ is arbitrary then the function $g$ has a stochastic extension in $L^{1}\left(B, \mu_{B, h}\right)$. For all $n$, one has,

$$
\begin{gathered}
\int_{B^{2}}(G \circ \chi)\left(\tilde{\pi}_{E_{n}}(Z)\right) d \mu_{B^{2}, h / 2}(Z)=\int_{E_{n}}(G \circ \chi)(Z) d \mu_{E_{n}, h / 2}(Z) \\
=\int_{\chi E_{n}} G(Z) d \mu_{\chi E_{n}, h / 2}(Z)=\int_{B^{2}} G\left(\tilde{\pi}_{\chi E_{n}}(Z)\right) d \mu_{B^{2}, h / 2}(Z) .
\end{gathered}
$$

We deduces (14) making $n$ going to infinity. 


\subsection{An Hilbertian basis of $H$.}

The set of pseudodifferential operators recalled in Section 3.1 is not invariant by a change of basis. We then have to choose a particular basis of $H$. Here $E$ denotes the set of $L^{2}$ vector fields on the unit sphere $S^{2}$, namely, the mappings $f=\left(f_{1}, f_{2}, f_{3}\right)$ on $S^{2}$, taking values in $\mathbb{R}^{3}$ and satisfying,

$$
\omega_{1} f_{1}(\omega)+\omega_{2} f_{2}(\omega)+\omega_{3} f_{3}(\omega)=0 \text { a. e. }
$$

This space is equipped with the norm

$$
\|f\|_{E}^{2}=\sum_{j=1}^{3} \int_{S^{2}}\left|f_{j}(\omega)\right|^{2} d \mu(\omega)
$$

where $\mu$ is natural measure on $S^{2}\left(\mu\left(S^{2}\right)=4 \pi\right)$. Thus $H$ is identified to $L^{2}\left(\mathbb{R}_{+}, r^{2} d r, E\right)$. We obtain an Hilbertian basis of $H$ written as,

$$
f_{m n}(k)=u_{m}(|k|) v_{n}(k /|k|)
$$

where $\left(u_{m}\right)$ is an Hilbertian basis of $L^{2}\left(\mathbb{R}_{+}, r^{2} d r\right)$ and where $\left(v_{n}\right)$ is an Hilbertian basis of $E$. We are then led to make a particular choice.

The space $E$ may be identified as the space of 1-differential forms on the unit sphere $S^{2}$, being in $L^{2}$ for the natural measure on this sphere. The de Rham Laplacian $\Delta_{S}$ is available on this space. One chooses the basis $v_{n}$ of $E$ as an Hilbertian basis of $E$ constituted with of eigenvectors of $\Delta_{S}$,

$$
\Delta_{S} v_{n}=\mu_{n} v_{n}
$$

The $\mu_{n}$ 's are the non decreasing sequence of eigenvalues of the de Rham Laplacian, repeated with their multiplicity. The function $u_{m}$ are the eigenvectors of the following operator,

$$
L=-\frac{d^{2}}{d r^{2}}-\frac{2}{r} \frac{d}{d r}+r^{2}
$$

being essentially selfadjoint in $L^{2}\left(\mathbb{R}_{+}, r^{2} d r\right)$ (see Reed-Simon [34], Theorem X.11). One checks that,

$$
L u(r)=\lambda u(r),
$$

setting $u(r)=e^{-\frac{r^{2}}{2}} \psi\left(r^{2}\right)$ and $x=r^{2}$, is equivalent to,

$$
x \psi^{\prime \prime}(x)=\left(\frac{3}{2}-x\right) \psi^{\prime}(x)+\frac{\lambda-3}{4} \psi(x)=0 .
$$

Thus, the eigenvalues of $L$ are the $\lambda_{m}=4 m+3(m \in \mathbb{N})$ of multiplicity 1 and the corresponding functions $\psi_{m}$ in (16) are the generalized Laguerre's polynomials $L_{m}^{(1 / 2)}$, up to a multiplicative factor. There exists 
an Hilbertian basis $\left(u_{m}\right)$ of $L^{2}\left(\mathbb{R}_{+}, r^{2} d r\right)$ constituted with eigenfunctions of $L$,

$$
L u_{m}=(3+4 m) u_{m}, \quad u_{m}(r)=C_{m} e^{-\frac{r^{2}}{2}} L_{m}^{(1 / 2)}\left(r^{2}\right) .
$$

When the Banach space $B$ is constructed as before, one sees that the $f_{m n}$ are elements of $B^{\prime}$. Indeed, the three components of $f_{m n}$ are in $\mathcal{S}\left(\mathbb{R}^{3}\right)$ and consequently, for all $\varphi$ in $H$,

$$
\left|\left(f_{m p}, \varphi\right)\right| \leq C_{m p}|D \varphi|
$$

where $D$ is defined in (12).

Proposition 2.4. For all mappings $\varphi=\left(\varphi_{1}, \varphi_{2}, \varphi_{3}\right)$ in $H$ with components in $\mathcal{S}\left(\mathbb{R}^{3}\right)$ and vanishing in a neighborhood of the origin, one has,

$$
m^{\alpha} n^{\beta}\left|\left(f_{m n} \cdot \varphi\right)\right| \leq \sum_{j=1}^{3} N_{\alpha \beta}\left(\varphi_{j}\right)
$$

where $N_{\alpha \beta}$ is a semi-norm on $\mathcal{S}\left(\mathbb{R}^{3}\right)$. In particular, the sequence $\left|\left(f_{m n}\right) \cdot \varphi\right|$ is summable.

Proof. One has,

$$
\lambda_{m}^{\alpha} \mu_{n}^{\beta}\left(f_{m n}\right) \cdot \varphi=\left(L^{\alpha} u_{m} \otimes \Delta_{S}^{\beta} v_{n}\right) \cdot \varphi=f_{m n} \cdot\left(\left(L^{\alpha} \otimes \Delta_{S}^{\beta}\right) \varphi\right) .
$$

If $\varphi$ belongs to $\mathcal{S}\left(\mathbb{R}^{3}, \mathbb{R}^{3}\right)$ and vanishes in a neighborhood of the origin, then integrating by parts gives,

$$
\lambda_{m}^{\alpha} \mu_{n}^{\beta}\left|\left(f_{m n}\right) \cdot \varphi\right| \leq\left\|\left(\left(L^{\alpha} \otimes \Delta_{S}^{\beta}\right) \varphi\right)\right\| .
$$

Since $m \leq \lambda_{m}=4 m+3$ and since the eigenvalues $\mu_{n}$ of the de Rham Laplacian satisfies $n \leq C \mu_{n}$ for some constant $C$ (see Ikeda-Tanigushi [26] or Folland [16]) then the proof of the Proposition is completed.

\section{Usual operators in QED.}

\subsection{Pseudodifferentials operators.}

Definition 3.1. Let $\mathcal{B}=\left(f_{j k}\right)$ be the Hilbertian basis of $H$ defined in Section [2.3. We called multi-index $(\alpha, \beta)$ a map from $\mathbb{N}^{2}$ into $\mathbb{N} \times \mathbb{N}$ satisfying $\alpha_{j k}=\beta_{j k}=0$ for all but a finite number of indices. For all integers $m \geq 0, \mathcal{M}_{m}$ denotes the set of multi-indices $(\alpha, \beta)$ satisfying $\alpha_{j k} \leq m$ and $\beta_{j k} \leq m$, for all 
$(j, k)$. For any integer $m \geq 0$, any $M>0$, and for each summable sequence of nonnegative real numbers $\varepsilon=\left(\varepsilon_{j k}\right)$, we denote by $S_{m}(\mathcal{B}, M, \varepsilon)$ the set of continuous functions $F$ from $H^{2}$ to $\mathbb{C}$ such that, for all multi-indices $(\alpha, \beta)$ in $\mathcal{M}_{m}$, the derivative $\partial_{q}^{\alpha} \partial_{p}^{\beta} F$ is well defined, continuous and bounded, and satisfies

$$
\left|\partial_{q}^{\alpha} \partial_{p}^{\beta} F(q, p)\right| \leq M \prod \varepsilon_{j k}^{\alpha_{j k}+\beta_{j k}} .
$$

We set $S_{m}(\mathcal{B}, \varepsilon)=\bigcup_{M \geq 0} S_{m}(\mathcal{B}, M, \varepsilon)$. For all $F \in S_{m}(\varepsilon)$, we set $\|F\|_{m, \varepsilon}=\inf \{M \geq 0: F \in$ $\left.S_{m}(\mathcal{B}, M, \varepsilon)\right\}$. If $F$ depends on one or several parameters, we say that $F$ is bounded in $S_{m}(\mathcal{B}, \varepsilon)$ if the norm $\|F\|_{m, \varepsilon}$ is bounded independently of these parameters.

We denote by $S_{\infty}(\mathcal{B}, \varepsilon)$ the intersection of the classes $S_{m}(\mathcal{B}, \varepsilon)$. We say that $F$, depending on some parameters, is bounded in $S_{\infty}(\mathcal{B}, \varepsilon)$ if, for all $m$, the norm $\|F\|_{m, \varepsilon}$ is bounded independently of these parameters (but usually not on $\mathrm{m}$ ).

We denote by $S_{\infty}^{\text {mat }}(\mathcal{B}, \varepsilon)$ the analogous space for mappings taking values in $\mathcal{L}\left(\left(\mathbb{C}^{2}\right)^{\otimes N}\right)$.

The Weyl calculus in infinite dimension [3] and [4] enables to associate quadratic forms on the space $\mathcal{D}$ of Definition 2.2 with suitable functions. It is sufficient that the function satisfies hypotheses $\left(H_{1}\right)$ and $\left(H_{2}\right)$ of [4] (Section 1). The construction of the quadratic form is given in [3] and, in a simpler way, in [4] (Theorem 2.2). If the function $F$ belongs to $S_{2}(\mathcal{B}, \varepsilon)$, and if the sequence $\varepsilon_{m n}$ is summable then hypotheses $\left(H_{1}\right)$ and $\left(H_{2}\right)$ of [4] are satisfied. In this case, one shows in Theorem 1.4 in [3], using Proposition 8.4 in [3], that the quadratic form initially defined on $\mathcal{D}$ is the quadratic form of a bounded operator in $L^{2}\left(B, \mu_{B, h / 2}\right)$ denoted $O p_{h}^{\text {weyl }}(F)$.

Besides the sets $S_{2}(\mathcal{B}, \varepsilon)$, there is another important class of functions satisfying hypotheses $\left(H_{1}\right)$ and $\left(H_{2}\right)$ of [4] and to which we may associate a quadratic form on $\mathcal{D}$. These are symbols $L(q, p)$ which are continuous linear forms on $H^{2}$. One shows (4], Proposition 2.4) that the Weyl quadratic form associated with such a symbol is the one of an operator from $\mathcal{D}$ to $\mathcal{D}$, also denoted $O p_{h}^{\text {weyl }}(L)$. We may write $L(q, p)=(a, q)+(b, p)$, with $a$ and $b$ in $H$. The Weyl quantized operator $O p_{h}^{\text {weyl }}(L)$ associated with this function is usually denoted $\Phi_{S}(a+i b)$ and called Segal field. Namely, using the canonical Segal isomorphism $\mathcal{J}_{h}: \mathcal{F}_{s}\left(H_{\mathbb{C}}\right) \rightarrow L^{2}\left(B, \mu_{B, h / 2}\right)$, this operator becomes,

$$
\mathcal{J}_{h}^{-1} O p_{h}^{\text {weyl }}(L) \mathcal{J}_{h}=\sqrt{h} \Phi_{S}(a+i b)
$$

where the Segal field $\Phi_{S}(a+i b)$ is defined in [34]. It may be defined without pseudodifferential formalism on Fock space, using creation and annihilation operators. For any $a$ in $H$, we denote by $Q_{h}(a)$ and $P_{h}(a)$ the operators associated with the functions $(q, p) \mapsto(a, q)$ and $(q, p) \mapsto(a, p)$ by the mapping $O p_{h}^{\text {weyl }}$. 
For any $X=(a, b)$ in $H^{2}$ and for each $h>0, \Psi_{X, h}$ denotes the corresponding coherent state being an element of $\mathcal{F}_{s}\left(H_{\mathbb{C}}\right)$ defined by,

$$
\Psi_{(a, b), h}=\sum_{n \geq 0} \frac{e^{-\frac{|a|^{2}+|b|^{2}}{4 h}}}{(2 h)^{n / 2} \sqrt{n !}}(a+i b) \otimes \cdots \otimes(a+i b) .
$$

In the space $L^{2}\left(B, \mu_{B, h / 2}\right)$, the coherent state is also denoted by $\Psi_{(a, b), h}$ and is defined by ([4]),

$$
\Psi_{a, b, h}(u)=e^{\frac{1}{h} \ell_{(a+i b)}(u)-\frac{1}{2 h}|a|^{2}-\frac{i}{2 h} a \cdot b}, \quad \text { a.e. } u \in B .
$$

See Section 2.2 for $\ell_{(a+i b)}$.

Proposition 3.2. If $F$ belongs to $S_{\infty}(M, \varepsilon)$ and $G$ to $S_{\infty}\left(M^{\prime}, \delta\right)$ then the (possibly matricial) product $F G$ lies in $S_{\infty}\left(M M^{\prime}, \varepsilon+\delta\right)$. If $F$ takes scalar values, if $L$ is a continuous linear form on $H^{2}$ and if the sequence $\left(\varepsilon_{m n}\right)$ is square summable, then the Poisson bracket $\{F, L\}$ lies in $S_{\infty}\left(M^{\prime \prime}, \varepsilon\right)$ with,

$$
M^{\prime \prime}=\sum_{m n} \varepsilon_{m n}\left(\left|L\left(f_{m n}, 0\right)\right|+\left|L\left(0, f_{m n}\right)\right|\right)
$$

With a given quadratic form $Q$ on the space $\mathcal{D}$, one may define its Wick symbol by,

$$
\sigma_{h}^{\text {wick }}(Q)(X)=Q\left(\Psi_{X, h}, \Psi_{X, h}\right), \quad X \in H^{2}
$$

where the $\Psi_{X, h}$ are coherent states whose definition is reminded in (16) or in (17) of [4. One also defines the bi-symbol by,

$$
\left(S_{h} Q\right)(X, Y)=\frac{Q\left(\Psi_{X, h}, \Psi_{Y, h}\right)}{<\Psi_{X, h}, \Psi_{Y, h}>}, \quad(X, Y) \in H^{2} \times H^{2} .
$$

One similarly defines $\sigma_{h}^{\text {wick }}(A)$ and $S_{h} A$ when $A$ is an operator from $\mathcal{D}$ to $L^{2}\left(B, \mu_{B, h / 2}\right)$.

\subsection{The functor $\Gamma$ and $\mathrm{d} \Gamma$. Photons number and energy.}

Let $T$ be a selfadjoint operator, bounded or unbounded with domain $D(T)$ in $H^{2}$ and being $\mathbb{C}$-linear when $H^{2}$ and $H_{\mathbb{C}}$ are identified (that is to say, when the operator $T$ commutes with the map $\mathcal{F}$ defined by $\mathcal{F}(q, p)=(-p, q))$. One sets, for any finite sequence $\left(u_{1}, . . u_{m}\right)$ in $D(T)$,

$$
d \Gamma(T)\left(u_{1} \otimes \cdots \otimes u_{m}\right)=\left(T u_{1}\right) \otimes \cdots \otimes u_{m}+\cdots+u_{1} \otimes \cdots \otimes\left(T u_{m}\right) .
$$

By linearity, one defines an operator on the subspace of $\mathcal{F}_{s}\left(H_{\mathbb{C}}\right)$ generated by this type of elements. One shows ([33]) that this operator is essentially selfadjoint and $\mathrm{d} \Gamma(T)$ also denotes its selfadjoint extension. 
Let $S$ be a selfadjoint operator, bounded in $H^{2}$, and commuting with $\mathcal{F}$. Then the Wick symbol of $\mathrm{d} \Gamma(S)$ is $F(z)=\left\langle S(z), z>/ 2 h\right.$ if we identify $(q, p) \in H^{2}$ with $q+i p \in H_{\mathbb{C}}$ (notation (5)). This property enables to consider $\mathrm{d} \Gamma(S)$ as a quadratic Hamiltonian of the type studied in [29] by other methods.

To any operator $M$, bounded in $H_{\mathbb{C}}$ with a norm smaller or equal than 1 , one associates an operator $\Gamma(M)$, bounded in $\mathcal{F}_{s}\left(H_{\mathbb{C}}\right)$, such that, for all $u_{1}, \ldots, u_{m}$ in $H_{\mathbb{C}}$, one has,

$$
\Gamma(M)\left(u_{1} \otimes \ldots \otimes u_{m}\right)=\left(M u_{1}\right) \otimes \ldots \otimes\left(M u_{m}\right) .
$$

In view of the usual isomorphism, these definitions are moved to the space $L^{2}\left(B, \mu_{B, h / 2}\right)$.

When $T=I$, the operator $N=\mathrm{d} \Gamma(I)$ is the number operator. When $T=h M_{\omega}$, where $M_{\omega}$ is the multiplication, in $H_{\mathbb{C}}$ or in $H^{2}$, by $\omega(k)=|k|$, the operator $\mathrm{d} \Gamma\left(h M_{\omega}\right)$, denoted $H_{p h}$, is the photons Hamiltonian. If $M$ is the multiplication by $e^{i t h \omega(k)}$, then the operator $\Gamma(M)$ is unitary in $H_{\mathbb{C}}$ and is precisely $e^{i t H_{p h}}$. For any rotation $R$ in $S O(3)$, one may define an operator $\pi(R)$ in $H$, and then an operator $\Pi(R)$ in $\mathcal{H}_{p h}$ by,

$$
(\pi(R) f)(k)=R f\left(R^{-1} k\right), \quad \Pi(R)=\Gamma(\pi(R)) .
$$

Thus, one defines a unitary representation of $S O(3)$ in $\mathcal{H}_{p h}$ which is used in Section 4

In the literature, the operator $O p_{h}^{\text {weyl }}\left(F_{a b}\right)$ is usually often denoted by $h^{1 / 2} \Phi_{S}(a+i b)$, where $F_{a b}$ is the linear form on $H^{2}$ defined by $F_{a b}(q, p)=a \cdot q+b \cdot p$, with $(a, b) \in H^{2}$.

We denote the domain of the operator $N^{m / 2}$ by $W_{m}$ (Sobolev space) and it is equipped with,

$$
\|u\|_{W_{m}}^{2}=<(I+2 h N)^{m} u, u>.
$$

In particular, for $m=1$, one has,

$$
\|u\|_{W_{1}}^{2}=\|u\|^{2}+\sum_{(m, n) \in \Gamma}\left\|\left(Q_{h}\left(f_{m n}\right)+i P_{h}\left(f_{m n}\right)\right) u\right\|^{2} .
$$

One knows that, when $L$ is a continuous linear form on $H^{2}$, the operator $O p_{h}^{\text {weyl }}(L)$ initially defined from $\mathcal{D}$ into $\mathcal{D}$, is extended as an operator from $W_{1}$ to $L^{2}\left(B, \mu_{B, h / 2}\right)$. See Lemma 2.3 in 13 . One also shows in Proposition 2.8 of [4] that, if $F$ belongs to $S_{\infty}(\mathcal{B}, \varepsilon)$ with a summable sequence $\left(\varepsilon_{m n}\right)$, then the operator $O p_{h}^{\text {weyl }}(F)$ is also bounded in $W_{m}$.

The following Proposition underlines the relation between the functor $\Gamma$ and analogue of metaplectic operators, and between $\mathrm{d} \Gamma$ and quadratic Hamiltonians. See Dereziński-Gerard Lemmas 2.1 and 2.3 of 
13] for an analogous statement. See B. Lascar [29] for an analogue of the metaplectic group in infinite dimension. See Combescure Robert [12] for quadratic Hamiltonians in finite dimension.

Proposition 3.3. Let $T$ be an unitary map in $H^{2}$ being $\mathbb{C}$-linear when identifying $H^{2}$ and $H_{\mathbb{C}}$ (in other words, commuting with the map $\mathcal{F}$ defined by $\mathcal{F}(q, p)=(-p, q)$ ). (These hypotheses imply that $T$ is symplectic). For any $X$ in $H^{2}$, one has,

$$
\Gamma(T) \Psi_{X, h}=\Psi_{T X, h},
$$

where the $\Psi_{X, h}$ are coherent states. The Wick symbol of the operator $\Gamma(T)$ is, with notation (5) and identifying $X=(q, p) \in H^{2}$ with $z=q+i p \in H_{\mathbb{C}}$,

$$
<\Gamma(T) \Psi_{z, h}, \Psi_{z, h}>=e^{-\frac{1}{2 h}|z|^{2}+\frac{1}{2 h}<T z, z>} .
$$

Let $\Phi$ be a continuous linear form on $H^{2}$. One has,

$$
(\Gamma(T))^{-1} O p_{h}^{\text {weyl }}(\Phi) \Gamma(T)=O p_{h}^{\text {weyl }}(\Phi \circ T) .
$$

Let $S$ be a bounded selfadjoint operator in $H^{2}$ commuting with $\mathcal{F}$, one has,

$$
\left[\mathrm{d} \Gamma(S), O p_{h}^{\text {weyl }}(\Phi)\right]=i O p_{h}^{\text {weyl }}(\Phi \circ S \circ \mathcal{F}) .
$$

This commutator is a priori defined as a mapping from $W_{3}$ into $L^{2}\left(B, \mu_{B, h / 2}\right)$.

If $S$ is unbounded then the result remains valid when the mapping $\Phi \circ S: D(S) \rightarrow H^{2}$ is extended to a bounded operator in $H^{2}$.

The right hand side of (33) may be used to define a Poisson bracket.

We apply Proposition 3.3 with the map $T=\chi_{t}: H^{2} \rightarrow H^{2}$ which is identified to the multiplication by $e^{-i t|k|}$ when $H^{2}$ and $H_{\mathbb{C}}$ are identified. That is to say, with $\omega(k)=|k|$,

$$
\chi_{t}(q, p)=\left(q_{t}, p_{t}\right), \quad\left\{\begin{array}{l}
q_{t}(k)=\cos (t \omega(k)) q(k)+\sin (t \omega(k)) p(k) \\
p_{t}(k)=-\sin (t \omega(k)) q(k)+\cos (t \omega(k)) p(k)
\end{array} .\right.
$$

In view of Proposition 3.3

$$
e^{-i \frac{t}{h} H_{p h}} \Psi_{q, p, h}=\Gamma\left(\chi_{t}\right) \Psi_{q, p, h}=\Psi_{\chi_{t}(q, p), h} .
$$

We denote by $H^{\omega}$ the set of all $a$ in $H$ such that $|k| a(k)$ belongs to $H$ and by $H_{\omega}$ the set of all $a$ in $H$ such that $a(k) / \sqrt{|k|}$ lies in $H$. 
Proposition 3.4. i) The space $\mathcal{D} \cap D\left(H_{p h}\right)$ is dense in $W_{1}$ and in $D\left(H_{p h}\right)$.

ii) For all $a$ and $b$ in $H$, let $F_{a b}$ be the linear form on $H^{2}$ defined by $F_{a b}(q, p)=a \cdot q+b \cdot p$. Then, for all $a$ and $b$ in $H_{\omega}$, the operator $O p_{h}^{\text {weyl }}\left(F_{a, b}\right)$, initially defined in $\mathcal{D}$, is extended as continuous operator from $D\left(H_{p h}\right)$ in $L^{2}\left(B, \mu_{B, h / 2}\right)$.

Proof. i) For all finite sequences $a_{1}, \ldots, a_{n}$ in $H^{\omega}$, and for all polynomial functions $\Phi$, the function $f$ defined almost everywhere on $B$ by,

$$
f(x)=\Phi\left(\ell_{a_{1}}(x), \ldots, \ell_{a_{n}}(x)\right), \quad \text { a. e. } x \in B
$$

belongs to $\mathcal{D} \bigcap D\left(H_{p h}\right)$ and the space of this kind of functions is dense in $D\left(H_{p h}\right)$. Indeed, the set of functions $f$ defined in (36) is, using Segal isomorphism, the space spanned by symmetrized tensorial products $a_{1} \odot \cdots \odot a_{n}, a_{j} \in H^{\omega}$, which is a core of $H_{p h}$ ([34]). Let us verify that the set of functions written as (36) with the $a_{k}$ in $H$, is dense in $W_{1}$. Indeed, one knows that the norm of $W_{1}$ may also be written as, using the number operator $N$ or orthogonal projections $f_{n}$ of an element $f$ of the Fock space into $n$ photons spaces,

$$
\|f\|_{W_{1}}^{2}=<(I+2 h N) f, f>=\|f\|^{2}+2 h \sum_{n=1}^{\infty} n\left\|f_{n}\right\|^{2} .
$$

Consequently, for any $f$ in $W_{1}$, the sum $f_{0}+\cdots+f_{n}$ tends to $f$ in $W_{1}$. Via the Segal isomorphism, this amounts to say that the set of functions under the form (36), with the $a_{k}$ in $H$ instead of $H^{\omega}$, is dense in $W_{1}$. A function as in (36) is a finite linear combination of functions under the form $g=: \ell_{a_{1}} \ldots \ell_{a_{n}}$ : with the $a_{k}$ in $H$. One uses here the notation for the Wick product of the functions $\ell_{a_{k}}$, that is to say, the range by the Segal isomorphism of the symmetrized $a_{1} \odot \cdots \odot a_{n}\left([27)\right.$. Let $a_{k}^{(\nu)}$ be a sequence in $H^{\omega}$ converging to $a_{k}$ in $H$. Let $g^{\nu}=: \ell_{a_{1}^{\nu}} \ldots \ell_{a_{n}^{\nu}}$ :. The sequence of functions $g^{\nu}$ belongs to $\mathcal{D} \cap \operatorname{Dom}\left(H_{p h}\right)$ and the sequence $\left(g^{\nu}\right)$ tends to $g$ in $W_{1}$. Using the Segal isomorphism, this amounts to say that the sequence $a_{1}^{(\nu)} \odot \cdots \odot a_{n}^{(\nu)}$ tends to $a_{1} \odot \cdots \odot a_{n}$ in $W_{1}$, which is standard.

ii) We use the following well-known inequalities, for all $\varphi \in D\left(H_{p h}^{1 / 2}\right), a$ and $b$ in $H_{\omega}$,

$$
\left\|O p_{h}^{\text {weyl }}\left(F_{a b}\right) \varphi\right\| \leq C(|a / \sqrt{\omega}|+|b / \sqrt{\omega}|)|| H_{p h}^{1 / 2} \varphi\left\|+C h^{1 / 2}(|a|+|b|)\right\| \varphi \| .
$$

See [7. Consequently, for all $\varepsilon>0$, there exists $C_{\varepsilon}$ also depending on $a, b$ and $h$, such that,

$$
\left\|O p_{h}^{\text {weyl }}\left(F_{a b}\right) \varphi\right\| \leq \varepsilon\left\|H_{p h} \varphi\right\|+C_{\varepsilon}\|\varphi\|, \quad \varphi \in D\left(H_{p h}\right) .
$$


Note that $e^{-i \frac{t}{h} H_{p h}}$ maps the space $\mathcal{D}$ of Definition 2.2 into itself. Indeed, let $f$ belongs to $\mathcal{D}_{E}$ where $E$ is finite $n$-dimensional subspace of $H$. The function $X \mapsto<f, \Psi_{X h}>$ lies in $L^{1}\left(E^{2}, \lambda\right)$ where $\lambda$ is the Lebesgue measure and we may write the vector values integral,

$$
f=(2 \pi h)^{-n} \int_{E^{2}}<f, \Psi_{X h}>\Psi_{X h} d \lambda(X) .
$$

Consequently, from (35),

$$
e^{-i \frac{t}{h} H_{p h}} f=(2 \pi h)^{-n} \int_{E^{2}}<f, \Psi_{X h}>\Psi_{\chi_{t}(X), h} d \lambda(X) .
$$

Let $F_{1}$ and $F_{2}$ be the projections of the space $\chi_{t}\left(E^{2}\right)$ on the two components of $H^{2}$. One sees that $e^{-i \frac{t}{h} H_{p h}} f$ belongs to $\mathcal{D}_{F_{1}+F_{2}}$.

Let $A$ be an operator from $\mathcal{D}$ into $\mathcal{D}$. One defines another operator from $\mathcal{D}$ in $\mathcal{D}$ by,

$$
A^{\text {free }}(t)=e^{i \frac{t}{h} H_{p h}} A e^{-i \frac{t}{h} H_{p h}},
$$

for all $t \in \mathbb{R}$. Then one has,

$$
\sigma_{h}^{\text {wick }}\left(A^{\text {free }}(t)\right)(q, p)=\sigma_{h}^{\text {wick }}(A)\left(\chi_{t}(q, p)\right)
$$

Indeed, for all $X$ in $H^{2}$,

$$
\left.<A^{\text {free }}(t) \Psi_{X h}, \Psi_{X h}>=<A e^{-i \frac{t}{h} H_{p h}} \Psi_{X h}, e^{-i \frac{t}{h} H_{p h}} \Psi_{X h}\right)=<A \Psi_{\chi_{t}(X), h}, \Psi_{\chi_{t}(X), h}>=\sigma_{h}^{w i c k}(A)\left(\chi_{t}(X)\right) .
$$

Note that the symplectic maps analogous to $\chi_{t}$ and the corresponding unitary operators are used by B. Lascar in Proposition 2.5 of 29 .

We now consider Weyl symbol.

Proposition 3.5. i) Let $F$ lying in $S_{2}(\mathcal{B}, \delta)$ where the sequence $\left(\delta_{m n}\right)$ is summable. Set $t \in \mathbb{R}$. Suppose that $F \circ \chi_{t}$ lies in $S_{2}(\mathcal{B}, \delta)$. Then, we have,

$$
O p_{h}^{\text {weyl }}\left(F \circ \chi_{t}\right)=e^{i \frac{t}{h} H_{p h}} O p_{h}^{\text {weyl }}(F) e^{-i \frac{t}{h} H_{p h}} .
$$

ii) Let $F$ and $G$ belong to $S_{2}(\mathcal{B}, \delta)$ where the sequence $\left(\delta_{m n}\right)$ is summable. Fix $t \in \mathbb{R}$ and assume that,

$$
O p_{h}^{w e y l}(G)=e^{i \frac{t}{h} H_{p h}} O p_{h}^{w e y l}(F) e^{-i \frac{t}{h} H_{p h}} .
$$

Then $G=F \circ \chi_{t}$.

iii) If $F$ is a continuous linear form on $H^{2}$ then we have (40) for any $t \in \mathbb{R}$. 
Proof. i) Let $A_{1}$ and $A_{2}$ be the operators in the left and right hand sides of equality (40). Denote by $\Phi_{j}=S_{h}\left(A_{j}\right)$ their Wick bi-symbol defined in (24). For any symbol $G$, also denote by $\Phi_{G}$ the Wick bi-symbol of $O p_{h}^{\text {weyl }}(G)$ similarly defined. One then has $\Phi_{1}(X, Y)=\Phi_{F \circ \chi_{t}}(X, Y)$ and, from (30) and since $\chi_{t}$ is unitary, $\Phi_{2}(X, Y)=\Phi_{F}\left(\chi_{t}(X), \chi_{t}(Y)\right)$, for $(X, Y) \in H^{2}$. In view of the definition in Theorem 2.2 of [4, with functions $\ell$ defined in Section 2.2 and the notion of stochastic extension in Definition 4.4 of 3 . recalled in Section 2.2 ,

$$
\Phi_{F}(X, Y)=\int_{B^{2}} \widetilde{F}(Z) e^{\frac{1}{h}\left(\ell_{X}(\bar{Z})+\ell_{\bar{Y}}(Z)-<X, Y>\right.} d \mu_{B^{2}, h / 2}(Z), \quad(X, Y) \in\left(H^{2} \times H^{2}\right)
$$

where $\widetilde{F}$ denotes the stochastic extension of $F$ which exists from Proposition 8.4 of [3]. In the above exponent, we have identified $H^{2}$ with $H_{\mathbb{C}}$ and the notation $<,>$ is the one of (5). Let us check that $\Phi_{1}=\Phi_{2}$ on $H^{2}$. To this end, set for all $X, Y$ and $Z$ in $H^{2}$,

$$
G_{2}(Z)=F(Z) e^{\frac{1}{h}\left(<\chi_{t}(X), Z>+<Z, \chi_{t}(Y)>\right)-<\chi_{t}(X), \chi_{t}(Y)>}
$$

and $G_{1}(Z)=G_{2}\left(\chi_{t}(Z)\right)$. These two functions of $Z$ have stochastic extensions $\widetilde{G}_{2}$ and $\widetilde{G}_{1}=\widetilde{G_{2} \circ \chi_{t}}$ in $L^{1}\left(B^{2}, \mu_{B^{2}, h / 2}\right)$. One has, for all $X$ and $Y$ in $H^{2}$, since $\chi_{t}$ is unitary

$$
\Phi_{2}(X, Y)=\int_{B^{2}} \widetilde{G}_{2}(Z) d \mu_{B^{2}, h / 2}(Z), \quad \Phi_{1}(X, Y)=\int_{B^{2}} \widetilde{G_{2} \circ \chi_{t}}(Z) d \mu_{B^{2}, h / 2}(Z) .
$$

These integrals are equal from Proposition 2.3. Consequently, one indeed has $\Phi_{1}=\Phi_{2}$ on $H^{2}$. Therefore, these two operators $A_{1}$ and $A_{2}$ have the same Wick bi-symbol. One concludes according to Lemma 2.7 of [4].

ii) Since $F$ and $G$ lies in $S_{2}(\mathcal{B}, \delta)$ then they have stochastic extensions $\widetilde{F}$ and $\widetilde{G}$ in $L^{2}\left(B, \mu_{B, h / 2}\right)$. From Proposition 2.3, this holds true for $F \circ \chi_{t}$, and $F \circ \chi_{t}$ satisfies hypotheses $\left(H_{1}\right)$ and $\left(H_{2}\right)$ in Theorem 2.2 of [4]. Then, one may associate a quadratic form $Q_{h}^{\text {weyl }}\left(F \circ \chi_{t}\right)$, and a priori not an operator, with $F \circ \chi_{t}$, and similarly for $G$. The same method as in i) shows that $Q_{h}^{\text {weyl }}\left(G-F \circ \chi_{t}\right)(f, g)=0$ for $(f, g) \in \mathcal{D}^{2}$. This implies that $\left(G-F \circ \chi_{t}\right) \circ P_{E}$ is vanishing when choosing $(f, g) \in \mathcal{D}_{E}^{2}$, for all vectors subspaces $E$ in $H$, and therefore that $G-F \circ \chi_{t}=0$.

iii) If $F$ is a continuous linear form on $H^{2}$ then this also true for $F \circ \chi_{t}$. The functions $F$ and $F \circ \chi_{t}$ remain fixed when applying the operator $H_{h / 2}$ defined in (37) of [4]. Consequently, from the equality (38) of 4, Weyl and Wick symbols of these operators are equal. Point iii) then follows from (33). 


\subsection{Field operators.}

For $x$ in $\mathbb{R}^{3}$, we now recall the standard definitions of the six operators $B_{j}(x)$ and $E_{j}(x)(1 \leq j \leq 3)$, unbounded in the space $\mathcal{H}_{p h}$, corresponding to the three components of the magnetic field and to the three components of the electric field. These operators are usually defined with the Segal fields formalism (see, e.g., [17] [18] [38]). However, to use pseudodifferential calculus, we associate these operators with symbols noted $B_{j}(x, q, p)$ and $E_{j}(x, q, p)$ which are linear in $(q, p)$. To define symbols $B_{j}(x, q, p)$, one chooses a function $\chi$ in $\mathcal{S}(\mathbb{R})$ vanishing in a neighborhood of 0 and set,

$$
B_{j, x}(k)=\frac{i \chi(|k|)|k|^{\frac{1}{2}}}{(2 \pi)^{\frac{3}{2}}} e^{-i k \cdot x} \frac{k \wedge e_{j}}{|k|}, \quad k \in \mathbb{R}^{3} \backslash\{0\}
$$

where $\left(e_{1}, e_{2}, e_{3}\right)$ is the canonical basis of $\mathbb{R}^{3}$.

The function $\chi$ is assumed to vanish in a neighborhood of the origin in order to apply the results in [3] and [4] in their current forms. A possible improvement of these results could allow to avoid this hypothesis.

Next, we set,

$$
B_{j}(x, q, p)=\left(\operatorname{Re} B_{j, x}, q\right)+\left(\operatorname{Im} B_{j, x}, p\right) .
$$

Explicitly, one has for instance,

$$
\begin{aligned}
B_{3}^{f r e e}(x, t, q, p)= & (2 \pi)^{-\frac{3}{2}} \int_{\mathbb{R}^{3}} \chi(|k|)|k|^{\frac{1}{2}}\left[-\sin (k \cdot x-t|k|)\left(k_{1} q_{2}(k)-k_{2} q_{1}(k)\right)\right. \\
& \left.-\cos (k \cdot x-t|k|)\left(k_{1} p_{2}(k)-k_{2} p_{1}(k)\right)\right] \frac{d k}{|k|}
\end{aligned}
$$

We denote by $B_{j}(x)$ the unbounded operator whose symbol is $B_{j}(x, q, p)$.

We also use the helicity operator $J$ from $H^{2}$ to $H^{2}$ and defined by,

$$
J(q, p)(k)=\left(\frac{k \wedge q(k)}{|k|}, \frac{k \wedge p(k)}{|k|}\right), \quad k \in \mathbb{R}^{3} \backslash\{0\} .
$$

One then sets,

$$
E_{j}(x, q, p)=-B_{j}(x, J(q, p))
$$

We use the commutation relations below, where we set,

$$
\rho(x)=(2 \pi)^{-3} \int_{\mathbb{R}^{3}}|\chi(k)|^{2} \cos (k \cdot x) d k .
$$


One has,

$$
\left[E_{1}(x), B_{2}(y)\right]=i h\left(\partial_{3} \rho\right)(y-x) .
$$

The other commutators follow by anti-symmetry and circular permutations. Also, $\left[E_{j}(x), B_{j}(y)\right]=0$. Moreover,

$$
\left[B_{j}(x), B_{m}(y)\right]=\left[E_{j}(x), E_{m}(y)\right]=0 .
$$

Indeed, if $A$ and $B$ are two operators with linear symbols $F$ and $G$ (two Segal fields) then one knows that the commutator $[A, B]$ is equal to the constant $(h / i)\{F, G\}$ where $\{F, G\}$ is the Poisson bracket. When $F(q, p)=(a, q)+(b, p)$ and $G(q, p)=\left(a^{\prime}, q\right)+\left(b^{\prime}, p\right)$, one has $\{F, G\}=\left(b, a^{\prime}\right)-\left(a, b^{\prime}\right)$.

Next, the fields free evolution operators are defined by,

$$
B_{j}^{f r e e}(x, t)=e^{i \frac{t}{h} H_{p h}} B_{j}(x) e^{-i \frac{t}{h} H_{p h}}, \quad E_{j}^{f r e e}(x, t)=e^{i \frac{t}{h} H_{p h}} E_{j}(x) e^{-i \frac{t}{h} H_{p h}} .
$$

From Proposition 3.4 (point iii), these operators have Weyl and Wick symbols,

$$
B_{j}^{\text {free }}(x, t, q, p)=B_{j}\left(x, \chi_{t}(q, p)\right), \quad E_{j}^{\text {free }}(x, t, q, p)=E_{j}\left(x, \chi_{t}(q, p)\right) .
$$

The operators valued vector fields $\mathbf{B}^{\text {free }}(x, t)=\left(B_{1}^{\text {free }}(x, t), B_{2}^{\text {free }}(x, t), B_{3}^{\text {free }}(x, t)\right)$ and $\mathbf{E}^{\text {free }}(x, t)$ satisfy Maxwell equations in vacuum,

$$
\begin{gathered}
\operatorname{div} \mathbf{B}^{\text {free }}(x, t)=\operatorname{div} \mathbf{E}^{\text {free }}(x, t)=0 \\
\frac{d}{d t} \mathbf{B}^{\text {free }}(x, t)=-\operatorname{rot} \mathbf{E}^{\text {free }}(x, t), \quad \frac{d}{d t} \mathbf{E}^{\text {free }}(x, t)=\operatorname{rot} \mathbf{B}^{\text {free }}(x, t) .
\end{gathered}
$$

The symbols of these operators satisfies the same equations.

\section{Spin-photon interaction model.}

The below model describing the quantized electromagnetic field interacting with $N$ fixed $1 / 2$ spin particles may be found in Reuse [35] (Section 4.11) in the case of one particle and then extended in a straightforward way for $N$ particles.

The Hilbert space of the quantized field is,

$$
\mathcal{H}_{p h}=L^{2}\left(B, \mu_{B, h / 2}\right) \simeq \mathcal{F}_{s}\left(H_{\mathbb{C}}\right)
$$


where $H$ is the Hilbert space of Section 2.1, $B$ and $\mu_{B, h / 2}$ are the Banach space and the measure defined in Section 2.2. In this space $\mathcal{H}_{p h}$, one considers the photons Hamiltonian,

$$
H_{p h}=h \mathrm{~d} \Gamma\left(M_{\omega}\right)
$$

( $M_{\omega}$ being the multiplication by $\left.|k|\right)$.

The Hilbert space of a $1 / 2$ spin particle, fixed at the origin, is $\mathbb{C}^{2}$. The Hamiltonian of this particle in an exterior magnetic field $\beta=\left(\beta_{1}, \beta_{2}, \beta_{3}\right) \in \mathbb{R}^{3}$ is,

$$
H_{m a g}=\sum_{j=1}^{3} \beta_{j} \sigma_{j}
$$

where the matrices $\sigma_{j}(1 \leq j \leq 3)$ are the Pauli matrices,

$$
\sigma_{1}=\left(\begin{array}{cc}
0 & 1 \\
1 & 0
\end{array}\right) \quad \sigma_{2}=\left(\begin{array}{cc}
0 & -i \\
i & 0
\end{array}\right) \quad \sigma_{3}=\left(\begin{array}{cc}
1 & 0 \\
0 & -1
\end{array}\right) .
$$

The Hilbert space of the entire system constituted with the photons field and with $N$ fixed $1 / 2$ spin particles at the points in $\mathbb{R}^{3}$ denoted $a_{1}, \ldots, a_{N}$ is $\mathcal{F}_{s}(H) \otimes\left(\mathbb{C}^{2}\right)^{\otimes N}$. The Hamiltonian of this whole system is,

$$
H(h)=H_{0}+h H_{\text {int }}, \quad H_{0}=H_{p h} \otimes I
$$

with,

$$
H_{\text {int }}=\sum_{\lambda=1}^{N} \sum_{j=1}^{3}\left(\beta_{j}+B_{j}\left(a_{\lambda}\right)\right) \otimes \sigma_{j}^{[\lambda]}
$$

where, for all operators $A$ belonging to $\mathcal{L}\left(\mathbb{C}^{2}\right), A^{[\lambda]}$ denotes $I \otimes \cdots A \cdots \otimes I$ with $A$ located at the $\lambda^{t h}$ position.

Proposition 4.1. The operator $H(h)$ above, of domain $D(H(h))=D\left(H_{0}\right)=D\left(H_{p h}\right) \otimes\left(\mathbb{C}^{2}\right)^{\otimes N}$, is selfadjoint. For all $x$ in $\mathbb{R}^{3}$, the operators $B_{j}(x)$ and $E_{j}(x)(1 \leq j \leq 3)$ are bounded from $D(H(h))$ into $\mathcal{H}_{p h} \otimes\left(\mathbb{C}^{2}\right)^{\otimes N}$.

Indeed, from Proposition 3.4 point ii), one has for all $a$ and $b$ in the space $H_{\omega}$ defined before this Proposition, $D\left(H_{p h}\right) \subset D\left(O p_{h}^{\text {weyl }}\left(F_{a b}\right)\right)$ where $F_{a b}(q, p)=a \cdot q+b \cdot p$. Since the operators $B_{j}(0)$ are of this kind, one sees, for all $\phi \in D\left(H_{0}\right)$ and for any $\varepsilon>0$,

$$
\left\|H_{\text {int }} \phi\right\| \leq \varepsilon\left\|H_{0} \phi\right\|+C_{\varepsilon}\|\phi\|
$$

and one concludes applying Kato-Rellich Theorem. 
We can also consider the case of one $1 / 2$ spin particle, fixed at the origin, interacting both with a constant magnetic field and with a non quantized magnetic rotating field in an orthogonal plane to the constant field, and also interacting with the quantized field. Denoting the constant field $\beta=\left(0,0, \beta_{3}\right)$ and setting $\left(B_{1} \cos (\omega t), B_{1} \sin (\omega t), 0\right)$ as the rotating field then one gets the following Hamiltonian $H(h, t)$,

$$
H(h, t)=H(h)+h H_{\text {spin }}(t), \quad H_{\text {spin }}(t)=\left(B_{1} \cos (\omega t)\left(I \otimes \sigma_{1}\right)+B_{1} \sin (\omega t)\left(I \otimes \sigma_{2}\right)\right),
$$

where $H(h)$ is the Hamiltonian in (57) with $\beta=\left(0,0, \beta_{3}\right)$. The next Theorem verifies that, using a suitable transform, one may reduce this model to a time independent model with an Hamiltonian similar to the one in (57).

Theorem 4.2. One has,

$$
P(t)^{\star}\left(i h \frac{\partial}{\partial t}-H(h, t)\right) P(t)=i h \frac{\partial}{\partial t}-H_{T R}
$$

where

$$
H_{T R}=H_{0}+\sum_{j=1}^{3} B_{j}(0) \otimes \sigma_{j}+B_{1}\left(I \otimes \sigma_{1}\right)+\left(\beta_{3}-\frac{\omega}{2}\right)\left(I \otimes \sigma_{3}\right)
$$

and $P(t)=P_{p h}(t) \otimes P_{\text {spin }}(t)$ with $P_{p h}(t)$ and $P_{\text {spin }}(t)$ being operators in $\mathcal{H}_{p h}$ and $\mathbb{C}^{2}$ defined as following. One has, $\left.P_{p h}(t)=\Pi(R(-\omega t))\right)$ where $\Pi$ is the $S O(3)$ representation in $\mathcal{H}_{p h}$ defined in 27) and $R(\omega t)$ is the rotation with angle $\omega t$ in the horizontal plane. Moreover,

$$
P_{\text {spin }}(t)=\left(\begin{array}{cc}
\alpha(t) & 0 \\
0 & \alpha(-t)
\end{array}\right), \quad \alpha(t)=e^{-i \frac{\omega t}{2}} .
$$

Proof. Clearly, $P_{\text {spin }}(t)^{-1} \sigma_{j} P_{\text {spin }}(t)=\sigma_{j}(t)$ with,

$$
\sigma_{1}(t)=\left(\begin{array}{cc}
0 & e^{i \omega t} \\
e^{-i \omega t} & 0
\end{array}\right), \quad \sigma_{2}(t)=\left(\begin{array}{cc}
0 & -i e^{i \omega t} \\
i e^{-i \omega t} & 0
\end{array}\right), \quad \sigma_{3}(t)=\sigma_{3} .
$$

Consequently,

$$
\begin{gathered}
P_{\text {spin }}(t)^{-1}\left(\cos (\omega t) \sigma_{1}+\sin (\omega t) \sigma_{2}\right) P_{\text {spin }}(t)=\sigma_{1}, \\
P_{\text {spin }}(t)^{-1} \sigma_{3} P_{\text {spin }}(t)=\sigma_{3}, \\
P_{\text {spin }}(t)^{-1} i h \frac{\partial}{\partial t} P_{\text {spin }}(t)=i h \frac{\partial}{\partial t}+\frac{h}{2} \omega \sigma_{3} .
\end{gathered}
$$

Thus,

$$
\begin{aligned}
P_{\text {spin }}(t)^{-1}\left(i h \frac{\partial}{\partial t}\right. & \left.-h\left(B_{1} \cos (\omega t) \sigma_{1}+B_{1} \sin (\omega t) \sigma_{2}+\beta_{3} \sigma_{3}\right)\right) P_{\text {spin }}(t) \\
& =i h \frac{\partial}{\partial t}-h\left(B_{1} \sigma_{1}+\left(\beta_{3}-\frac{\omega}{2}\right) \sigma_{3}\right) .
\end{aligned}
$$


One knows that $H_{p h}=d \Gamma(h M)$ where $M$ is the multiplication by $|k|$ which commute with $U$ in such a way that $H_{p h}$ commutes with $\Gamma(U)$. Then,

$$
P_{p h}(t)^{-1} H_{p h} P_{p h}(t)=H_{p h}
$$

One now checks that,

$$
\sum_{j=1}^{3} P(t)^{-1}\left(B_{j}(0) \otimes \sigma_{j}\right) P(t)=\sum_{j=1}^{3} B_{j}(0) \otimes \sigma_{j} .
$$

From Proposition 3.3, one has,

$$
\sum_{j=1}^{3} P(t)^{-1}\left(B_{j}(0) \otimes \sigma_{j}\right) P(t)=\sum_{j=1}^{3} O p_{h}^{w e y l}\left(C_{j}(t, \cdot)\right) \otimes \sigma_{j}(t)
$$

where $\sigma_{j}(t)$ is defined in (64) and

$$
C_{j}(t, q, p)=B_{j}(0, U(t)(q, p))
$$

A direct computation shows that,

$$
C_{1}(t, \cdot)+i C_{2}(t, \cdot)=e^{i \omega t}\left(B_{1}(t, \cdot)+i B_{2}(t, \cdot)\right), \quad C_{1}(t, \cdot)-i C_{2}(t, \cdot)=e^{-i \omega t}\left(B_{1}(t, \cdot)-i B_{2}(t, \cdot)\right)
$$

and that $C_{3}(t, \cdot)=B_{3}(t, \cdot)$. Equality (67) is then obtained. Therefore, equality (61) follows from (65) (66) and (67).

\section{Proof of Theorem $1.1(i)(i i)(i v)$.}

We first begin by defining a numerical sequence $\left(\varepsilon_{m n}(t)\right)$ which, associated with the basis $\left(f_{m n}\right)$ in Section 2.3. plays a special role in our class of operators.

We set,

$$
H_{\text {int }}^{\text {free }}(t)=e^{i \frac{t}{h} H_{0}} H_{\text {int }} e^{-i \frac{t}{h} H_{0}}=\sum_{\lambda=1}^{N} \sum_{j=1}^{3}\left(\beta_{j}+B_{j}^{\text {free }}\left(a_{\lambda}, t\right) \otimes \sigma_{j}^{[\lambda]} .\right.
$$

We use the basis $\left(f_{m n}\right)$ defined in Section 2.3. The operators $P_{h}\left(f_{m n}\right)$ and $Q_{h}\left(f_{m n}\right)$ denote momentum and position operators corresponding to the element $f_{m n}$.

Proposition 5.1. Let $\left(f_{m n}\right)$ be the basis chosen in Section 2.3 and let $\chi$ be in $\mathcal{S}(\mathbb{R})$ vanishing in a neighborhood of the origin. Then there exists a sequence $\varepsilon_{m n}(t)$ satisfying,

$$
\left\|\left[P_{h}\left(f_{m n}\right), H_{i n t}^{\text {free }}(t)\right]\right\|+\left\|\left[Q_{h}\left(f_{m n}\right), H_{\text {int }}^{\text {free }}(t)\right]\right\| \leq h \varepsilon_{m n}(t) .
$$


Moreover, the sequence $\varepsilon_{m n}(t)$ is rapidly decreasing and in particular, it is summable, and $\varepsilon_{m n}(t)$ is a non decreasing function of $|t|$.

Proof. Set,

$$
B_{j x t}(k)=\frac{i \chi(|k|)|k|^{\frac{1}{2}}}{(2 \pi)^{\frac{3}{2}}} e^{i(t|k|-k \cdot x)} \frac{k \wedge e_{j}}{|k|}, \quad k \in \mathbb{R}^{3} \backslash\{0\} .
$$

The element $B_{j, a_{\lambda}, s}$ of $H$ has its three components belonging to $\mathcal{S}\left(\mathbb{R}^{3}\right)$ and theirs norms $N_{\alpha \beta}$ of the Proposition 2.4 are bounded by a constant $C_{\alpha \beta}(t)$ which is bounded on every compact set in $\mathbb{R}$. From Proposition 2.4. one sees,

$$
m^{\alpha} n^{\beta}\left|\left(B_{j, a_{\lambda}, s}, f_{m n}\right)\right| \leq C_{\alpha \beta}(t) .
$$

Thus, we may choose a function $\delta_{m n}(t)$ being a non decreasing function of $|t|$, which is summable for all $t$ and satisfying,

$$
\sup _{|s|<|t| \lambda \leq N} \sup _{\lambda \leq N}\left(\sup _{j \leq 3}\left|\left(B_{j, a_{\lambda}, s}, f_{m n}\right)\right|\right) \leq \delta_{m n}(t) .
$$

In view of (50) (34) (42) and (43), $B_{j}^{\text {free }}(x, t, q, p)$ is the scalar product of $B_{j x t}$ with $(q, p)$. According to the expression $H_{\text {int }}^{\text {free }}(t)$ in (68) together with (72), there exists a constant $K>0$ such that,

$$
\left\|\left[P_{h}\left(f_{m n}\right), H_{i n t}^{\text {free }}(t)\right]\right\|+\left\|\left[Q_{h}\left(f_{m n}\right), H_{i n t}^{\text {free }}(t)\right]\right\| \leq K h \delta_{m n}(t) .
$$

One therefore deduces the Proposition with $\varepsilon_{m n}(t)=K \delta_{m n}(t)$. Note that this family is summable from (71).

We now consider the following operator (interaction picture) defined by (1),

$$
U_{h}^{r e d}(t)=e^{i \frac{t}{h} H_{0}} e^{-i \frac{t}{h} H(h)} .
$$

In order to prove the pseudodifferential nature of $U_{h}^{\text {red }}(t)$, we now study iterated commutators of that operator with position $Q_{h}\left(f_{m n}\right)$ and momentum $P_{h}\left(f_{m n}\right)$ operators associated with the elements $f_{m n}$ of the basis $\mathcal{B}$ defined in Section 2.3 We prove that, these iterated commutators, a priori defined as quadratic forms on some subspaces, are extended as bounded operators in $\mathcal{H}_{p h} \otimes\left(\mathbb{C}^{2}\right)^{\otimes N}$. Let us start with simple commutators. For each continuous linear form $F$ on $H^{2}$, one defines the following commutator as a quadratic form on $W_{1} \otimes\left(\mathbb{C}^{2}\right)^{\otimes N}$ by,

$$
\begin{gathered}
{\left[O p_{h}^{\text {weyl }}(F) \otimes I, U_{h}^{\text {red }}(t)\right](f, g)=<U_{h}^{\text {red }}(t) f,\left(O p_{h}^{\text {weyl }}(F)^{\star} \otimes I\right) g>} \\
-<\left(O p_{h}^{\text {weyl }}(F) \otimes I\right) f, U_{h}^{r e d}(t)^{\star} g>.
\end{gathered}
$$


If $A_{1}, \ldots, A_{m}$ are operators, each being either one of the $P_{h}\left(f_{q r}\right) \otimes I$ or one of the $Q_{h}\left(f_{q r}\right) \otimes I$ associated with one of the element of the basis $\mathcal{B}$ (See sections 2.3 for this basis and 3.1 for position and momentum operators), one similarly defines the iterated commutator, with the notation $\operatorname{ad}(A) B=[A, B]$,

$$
\operatorname{ad}\left(A_{1}\right) \ldots \operatorname{ad}\left(A_{m}\right) U_{h}^{r e d}(t)
$$

being a priori defined as a bilinear form on $W_{m} \otimes\left(\mathbb{C}^{2}\right)^{\otimes N}$. Let us now prove that these commutators are extended as bounded operators in $\mathcal{H}_{p h} \otimes\left(\mathbb{C}^{2}\right)^{\otimes N}$. We begin with order one commutators.

Proposition 5.2. For all $f$ and $g$ in $W_{1} \otimes\left(\mathbb{C}^{2}\right)^{\otimes N}$, for any continuous linear form $F$ on $H^{2}$ and for all $t \in \mathbb{R}$, one has,

$$
\left[O p_{h}^{\text {weyl }}(F) \otimes I, U_{h}^{\text {red }}(t)\right](f, g)=<C(t) f, g>
$$

with,

$$
C(t) f=-i \int_{0}^{t} U_{h}^{r e d}(t) U_{h}^{r e d}(s)^{\star}\left[O p_{h}^{\text {weyl }}(F) \otimes I, H_{\text {int }}^{\text {free }}(s)\right] U_{h}^{\text {red }}(s) d s .
$$

Proof. First step. We denote by $H_{\omega}$ the set of all $q$ in $H$ such that the function $k \mapsto q(k) /|k|^{1 / 2}$ belongs to $H$. For any $a$ and $b$ in $H$, set $F_{a b}(q, p)=(a, q)+(b, p)$. We first prove equalities (76) and (77) when $f$ and $g$ lies in $\mathcal{D} \otimes\left(\mathbb{C}^{2}\right)^{\otimes N} \cap D(H(h))$ and when $F=F_{a b}$ with $a$ and $b$ in $H_{\omega}$. Set,

$$
Z(t)=U_{h}^{r e d}(t)^{\star}\left(O p_{h}^{\text {weyl }}\left(F_{a, b}\right) \otimes I\right) U_{h}^{\text {red }}(t) f .
$$

This indeed defines an element of $\mathcal{H}_{p h} \otimes\left(\mathbb{C}^{2}\right)^{\otimes N}$. Since $U_{h}^{\text {red }}(t)$ maps $D(H(h))$ into itself from (73) and Proposition 4.1, and since, from Proposition 3.4 for all $a$ and $b$ in $H_{\omega}$, the operator $O p_{h}^{\text {weyl }}\left(F_{a, b}\right) \otimes I$ maps $D(H(h))$ into $\mathcal{H}_{p h} \otimes\left(\mathbb{C}^{2}\right)^{\otimes N}$. From (42), $B_{j x}$ belongs to $H_{\omega}$, and $H_{\text {int }}$ maps $D(H(h))$ into $\mathcal{H}_{p h} \otimes\left(\mathbb{C}^{2}\right)^{\otimes N}$ and we have, for all $f$ in $D(H(h))$,

$$
\frac{d}{d t} U_{h}^{r e d}(t) f=-i H_{\text {int }}^{\text {free }}(t) U_{h}^{r e d}(t) f .
$$

Consequently, if $g$ is also in $D(H(h))$,

$$
\begin{gathered}
\frac{d}{d t}<Z(t), g>=-i<H_{\text {int }}^{\text {free }}(t) U_{h}^{\text {red }}(t) f,\left(O p_{h}^{\text {weyl }}\left(F_{a, b}\right)^{\star} \otimes I\right) U_{h}^{\text {red }}(t) g> \\
+i<\left(O p_{h}^{\text {weyl }}\left(F_{a, b}\right) \otimes I\right) U_{h}^{\text {red }}(t) f, H_{\text {int }}^{\text {free }}(t) U_{h}^{\text {red }}(t) g> \\
=-i<\left[\left(O p_{h}^{\text {weyl }}\left(F_{a, b}\right) \otimes I\right), H_{\text {int }}^{\text {free }}(t)\right] U_{h}^{\text {red }}(t) f, U_{h}^{\text {red }}(t) g>.
\end{gathered}
$$

The commutator $\left[O p_{h}^{\text {weyl }}\left(F_{a, b}\right) \otimes I, H_{\text {int }}^{\text {free }}(t)\right]$ is classically a constant matrix and then, is a bounded operator. We may then write,

$$
Z^{\prime}(t)=-i U_{h}^{\text {red }}(t)^{\star}\left[O p_{h}^{\text {weyl }}\left(F_{a, b}\right) \otimes I, H_{\text {int }}^{\text {free }}(t)\right] U_{h}^{\text {red }}(t) f .
$$


As a consequence, for all $f$ in $\left(\mathcal{D} \otimes\left(\mathbb{C}^{2}\right)^{\otimes N}\right) \bigcap D(H(h))$ and for and $a$ and $b$ in $H_{\omega}$, we have,

$$
U_{h}^{r e d}(t)^{\star} O p_{h}^{\text {weyl }}\left(F_{a, b}\right) U_{h}^{\text {red }}(t) f=O p_{h}^{\text {weyl }}\left(F_{a, b}\right) f-i \int_{0}^{t} U_{h}^{\text {red }}(s)^{\star}\left[O p_{h}^{\text {weyl }}\left(F_{a, b}\right), H_{\text {int }}^{\text {free }}(s)\right] U_{h}^{\text {red }}(s) f d s
$$

which implies, when acting $U_{h}^{r e d}(t)$ on the left side,

$$
\left[O p_{h}^{\text {weyl }}\left(F_{a, b}\right), U_{h}^{\text {red }}(t)\right] f=-i \int_{0}^{t} U_{h}^{r e d}(t) U_{h}^{r e d}(s)^{\star}\left[O p_{h}^{\text {weyl }}\left(F_{a, b}\right), H_{\text {int }}^{\text {free }}(s)\right] U_{h}^{\text {red }}(s) f d s .
$$

The Proposition is then proved in this case.

Second step. For any $(a, b)$ in $H^{2}$, we choose a sequence $\left(a_{j}, b_{j}\right)$ in $H_{\omega}^{2}$ converging to $(a, b)$ in $H^{2}$. From a standard result recalled in Proposition 2.8 of [4], for all $f$ in $W_{1} \otimes\left(\mathbb{C}^{2}\right)^{\otimes N}$,

$$
\left\|O p_{h}^{\text {weyl }}\left(F_{a, b}-F_{a_{j}, b_{j}}\right) f\right\| \leq C\left(\left|a-a_{j}\right|+\left|b-b_{j}\right|\right)\|f\|_{W_{1}} .
$$

Consequently,

$$
\lim _{j \rightarrow \infty}\left\|O p_{h}^{w e y l}\left(F_{a, b}-F_{a_{j}, b_{j}}\right) f\right\|=0, \quad \lim _{j \rightarrow \infty}\left\|\left[\left(F_{a, b}-F_{a_{j}, b_{j}}\right) \otimes I, H_{\text {int }}^{\text {free }}(t, \cdot)\right]\right\|=0
$$

Therefore, the Proposition holds true for all $f$ and $g$ in $\left(\mathcal{D} \otimes\left(\mathbf{C}^{2}\right)^{\otimes N}\right) \cap D(H(h)) \subset W_{1} \otimes\left(\mathbb{C}^{2}\right)^{\otimes N}$, and for any $a$ and $b$ in $H$.

Third step. Taking into account Proposition 3.4 the space $\left(\mathcal{D} \otimes\left(\mathbb{C}^{2}\right)^{\otimes N}\right) \bigcap D(H(h))$ is dense in $W_{1} \otimes$ $\left(\mathbb{C}^{2}\right)^{\otimes N}$ and the Proposition then follows.

We now turn to iterated commutators. For all $(m, n)$, we set $\widetilde{P}_{h}\left(f_{m n}\right)=P_{h}\left(f_{m n}\right) \otimes I$ and for all multiindex $(\alpha, \beta)$ we define a quadratic form on $\mathcal{D} \otimes\left(\mathbb{C}^{2}\right)^{\otimes N}$ denoted $\left(\operatorname{ad} \widetilde{P}_{h}\right)^{\alpha}\left(\operatorname{ad} \widetilde{Q}_{h}\right)^{\beta} U_{h}^{\text {red }}(t)$, as in Section 1 of 4 .

Proposition 5.3. Let $U_{h}^{\text {red }}(t)$ be the operator defined in (1) or 773). Then, for all multi-index $(\alpha, \beta)$, the quadratic form $\left(\operatorname{ad} \widetilde{P}_{h}\right)^{\alpha}\left(\operatorname{ad} \widetilde{Q}_{h}\right)^{\beta} U_{h}^{\text {red }}(t)$ is the quadratic form of a bounded operator, written with the same notation, which satisfies,

$$
\left\|\left(\operatorname{ad} \widetilde{P}_{h}\right)^{\alpha}\left(\operatorname{ad} \widetilde{Q}_{h}\right)^{\beta} U_{h}^{r e d}(t)\right\| \leq \prod_{m n}\left(h|t| \varepsilon_{m n}(t)\right)^{\alpha_{m n}+\beta_{m n}}
$$

where $\varepsilon_{m n}(t)$ is defined in Proposition 5.1 .

Proof. Let $A_{1}, \ldots, A_{n}$ be a finite sequence of operators, each operator $A_{j}$ being one of the $P_{h}\left(f_{q r}\right) \otimes I$ or one of the $Q_{h}\left(f_{q r}\right) \otimes I$ associated with one of the element of the basis $\mathcal{B}$, the corresponding indice $(q, r)$ 
being denoted $\psi(j)$. Iterating Propositon 5.2 , one gets,

$$
\begin{gathered}
\operatorname{ad} A_{1} \ldots \operatorname{ad} A_{n} U_{h}^{r e d}(t)=(-i)^{n} \sum_{\varphi \in S_{n}} \int_{\Delta_{n}(t)} U\left(t, s_{n}\right)\left[A_{\varphi(n)}, H_{\text {int }}^{\text {free }}\left(s_{n}\right)\right] U\left(s_{n}, s_{n-1}\right)\left[A_{\varphi(n-1)}, H_{\text {int }}^{\text {free }}\left(s_{n-1}\right)\right] \\
U\left(s_{2}, s_{1}\right)\left[A_{\varphi(1)}, H_{\text {int }}^{\text {free }}\left(s_{1}\right)\right] U\left(s_{1}, 0\right) d s_{1} \ldots d s_{n}
\end{gathered}
$$

where we set $U(t, s)=U_{h}^{r e d}(t)\left(U_{h}^{r e d}(s)\right)^{\star}$, where $S_{n}$ is the set of bijections $\varphi$ in the set $\{1, \ldots, n\}$, and, if $t>0$

$$
\Delta_{n}(t)=\left\{\left(s_{1}, \ldots, s_{n}\right) \in \mathbb{R}^{n}, 0<s_{1}<\ldots<s_{n}<t\right\} .
$$

From Proposition 5.1 and since the $\varepsilon_{m n}(t)$ is non decreasing, we obtain that, if $|s|<|t|$,

$$
\left\|\left[A_{j}, H_{\text {int }}^{\text {free }}(s)\right]\right\| \leq h \varepsilon_{\psi(j)}(t) .
$$

Consequently, since $U(t, s)$ is unitary,

$$
\left\|\operatorname{ad} A_{1} \ldots \operatorname{ad} A_{n} U_{h}^{r e d}(t)\right\| \leq \sum_{\varphi \in S_{n}} \prod_{j=1}^{n} h \varepsilon_{\psi(\varphi(j))}(t) \int_{\Delta_{n}(t)} d s_{1} \ldots d s_{n}
$$

where $\Delta_{n}(t)$ is defined in (82). The last integral equals to $|t|^{n} / n !$. The factors in front this integral are all equal. Therefore,

$$
\left\|\operatorname{ad} A_{1} \ldots \operatorname{ad} A_{n} U(t)\right\| \leq|t|^{n} \prod_{j=1}^{n} h \varepsilon_{\psi(j)}(t) .
$$

With notations in Theorem 1.2 of [4, this amounts to the statement of Proposition 5.3.

The Proposition below ends the proof of the first two claims in Theorem 1.1. We make use of the notation $\{F, G\}$ for the Poisson bracket of two functions $F$ and $G$ on $H^{2}$ taking values in $\mathcal{L}\left(\left(\mathbb{C}^{2}\right)^{\otimes N}\right)$, the product in the Poisson bracket being a matricial product.

Proposition 5.4. i) For $0<h<1$, the operator $U_{h}^{\text {red }}(t)$ is of the form $O p_{h}^{\text {weyl }}(U(t, \cdot, h))$ where $U(t, \cdot, h)$ is matricial symbol (taking values in $\mathcal{L}\left(\left(\mathbb{C}^{2}\right)^{\otimes N}\right)$ ) which is $C^{\infty}$ on $H^{2}$ and which satisfies, for all multiindex $(\alpha, \beta)$,

$$
\left|\partial_{q}^{\alpha} \partial_{p}^{\beta} U(t, q, p, h)\right| \leq M(h, t) \prod_{m n}\left(|t| \varepsilon_{m n}(t)\right)^{\left|\alpha_{m n}+\beta_{m n}\right|}
$$

where $\varepsilon_{m n}(t)$ is the sequence of the Proposition 5.1 and

$$
M(h, t)=\prod_{m n}\left(1+K S^{2} h|t|^{2} \varepsilon_{m n}(t)^{2}\right)
$$


with $K$ being a universal constant and

$$
S=\sup _{m n} \max \left(1,|t| \varepsilon_{m n}(t)\right) .
$$

In particular, the function $U(t, \cdot, h)$ belongs to $S_{\infty}^{\text {mat }}(\mathcal{B},|t| \varepsilon(t))$.

ii) Denoting by $H_{\text {int }}^{\text {free }}(t, q, p)$ the Weyl (matricial) symbol of $H_{\text {int }}^{\text {free }}(t)$ (defined in (68)), we have,

$$
\frac{d}{d t} U(t, q, p, h)=-i H_{\text {int }}^{f r e e}(t, q, p) U(t, q, p, h)-\frac{h}{2}\left\{H_{\text {int }}^{\text {free }}(t, \cdot), U(t, \cdot, h)\right\}(q, p) .
$$

The constant $K$ in (84) is related to the Weyl calculus but not to the physical system.

Proof of i) One first applies Theorem 1.2 of [4 with $A_{h}$ being components of the matricial operator $U_{h}^{r e d}(t)$ and $M=1, m=2$. The basis denoted $\left(e_{j}\right)$ in [4] here is the basis $\mathcal{B}=\left(f_{m n}\right)$ and the sequence $\left(\varepsilon_{j}\right)$ is $|t| \varepsilon_{m n}(t)$. From Proposition [5.3, the hypotheses (14) of this Theorem is fulfilled. From Theorem 1.2 of [4], there exists a matricial function $U(t, \cdot, h)$ whose components lies in $S_{2}(\mathcal{B},|t| \varepsilon(t))$ such that $U_{h}^{\text {red }}(t)=O p_{h}^{\text {weyl }}(U(t, \cdot, h))$. Moreover, this function satisfies (83) for $\alpha=\beta=0$. Next, for each multiindex $(\alpha, \beta)$, one applies Theorem 1.2 of $[4$ to the components of the following operator and constant $M$,

$$
A_{\alpha \beta}=\left(\operatorname{ad} \widetilde{P}_{h}\right)^{\alpha}\left(\operatorname{ad} \widetilde{Q}_{h}\right)^{\beta} U_{h}^{r e d}(t), \quad M=\prod_{m n}\left(h|t| \varepsilon_{m n}(t)\right)^{\left|\alpha_{m n}+\beta_{m n}\right|}
$$

with $\widetilde{P}_{h}\left(f_{m n}\right)=P_{h}\left(f_{m n}\right) \otimes I$. According to Theorem 1.2 of 4 , there exists a matricial function $U_{\alpha \beta}(t, \cdot, h)$ with components belonging to $S_{2}(\mathcal{B},|t| \varepsilon(t))$ such that $A_{\alpha \beta}=O p_{h}^{\text {weyl }}\left(U_{\alpha \beta}(t, \cdot, h)\right)$. Moreover, this function satisfies,

$$
\left|U_{\alpha \beta}(t, \cdot, h)\right| \leq M(h, t) \prod_{m n}\left(h|t| \varepsilon_{m n}(t)\right)^{\left|\alpha_{m n}+\beta_{m n}\right|} .
$$

Using symbols for the composition formulas that may be applied when one of the operators in the composition has its symbol defined as a continuous linear form on $H^{2}$ (see Proposition 2.6 of [4]),

$$
U_{\alpha \beta}(t, \cdot, h)=c_{\alpha \beta} h^{|\alpha+\beta|} \partial_{q}^{\alpha} \partial_{p}^{\beta} U(t, \cdot, h)
$$

with $\left|c_{\alpha \beta}\right|=1$. The proof is completed.

Proof of ii) According to point i) with Proposition 3.2 and Proposition 2.8 of [4], we can extend equality (178) for $f$ belonging to $W_{1} \otimes\left(\mathbb{C}^{2}\right)^{\otimes N}$. Consequently, for all $f$ and $g$ in $\mathcal{D} \otimes\left(\mathbb{C}^{2}\right)^{\otimes N}$,

$$
\frac{d}{d t}<U_{h}^{r e d}(t) f, g>=i<U_{h}^{r e d}(t) f, H_{\text {int }}^{\text {free }}(t) g>.
$$


The right hand side $\Phi_{t}(q, p, h)$ of (85) satisfies hypotheses $\left(H_{1}\right)$ and $\left(H_{2}\right)$ of $[4$. From Theorem 2.2 of [4], one then may associate a quadratic form $Q_{h}^{\text {weyl }}\left(\Phi_{t}\right)$ on $\mathcal{D}$ with it. In view of Proposition 2.6 in 4],

$$
-i<U_{h}^{r e d}(t) f, H_{\text {int }}^{\text {free }}(t) g>=Q_{h}^{\text {weyl }}\left(\Phi_{t}\right)(f, g) .
$$

Point ii) then follows.

Thus, the first two claims in Theorem 1.1 are derived. We next study the last claim.

For each $t \in \mathbb{R}$, the nonnegative quadratic form $g_{t}$ on $H^{2}$ is defined setting,

$$
g_{t}(X)=3 N|t| \sum_{m=1}^{3} \sum_{\lambda=1}^{N} \int_{0}^{t}\left|X \cdot B_{m, a_{\lambda}, s}\right|^{2} d s,
$$

where the $B_{m, a_{\lambda}, s}$ are elements of $H^{2}$ defined in (70) when identifying $H^{2}$ and $H_{\mathbb{C}}$.

Proposition 5.5. The matricial Weyl symbol $U(t, q, p, h)$ of the operator $U_{h}^{\text {red }}(t)$ is $C^{\infty}$ on $H^{2}$ and satisfies, for all integers $m$, for all vectors $X_{1}, \ldots, X_{m}$ in $H^{2}$, for all $t \in \mathbb{R}$,

$$
\left|d^{m} U(t, q, p, h)\left(X_{1}, \ldots, X_{m}\right)\right| \leq M(h, t) \prod_{j=1}^{m} g_{t}\left(X_{j}\right)^{1 / 2} \leq M(h, t)\left(\prod_{j=1}^{m} K t\left|X_{j}\right|\right)
$$

where $M(h, t)$ is defined in 84) and $K$ is a constant.

Proof. For any $t \in \mathbb{R}$ and for each $X \in H^{2}$, set

$$
N_{t}(X)=\sum_{m=1}^{3} \sum_{\lambda=1}^{N}\left|X \cdot B_{m, a_{\lambda}, t}\right| .
$$

Set, for any $X$ and $(q, p)$ in $H^{2}, F_{X}(q, p)=\sigma((q, p), X)$, where $\sigma$ is the symplectic form. For all $X$ in $H^{2}$ and all $t \in \mathbb{R}$, one has

$$
\left\|\left[O p_{h}^{\text {weyl }}\left(F_{X}\right), H_{\text {int }}^{\text {free }}(t)\right]\right\| \leq h N_{t}(X) .
$$

Equality (81) together with the above estimate show that, for all vectors $Y_{1}, \ldots, Y_{p}$ in $H^{2}$, setting $A_{Y_{j}}=$ $O p_{h}^{\text {weyl }}\left(F_{Y_{j}}\right) \otimes I$,

$$
\begin{gathered}
\left\|\operatorname{ad} A_{Y_{1}} \ldots \operatorname{ad} A_{Y_{p}} U_{h}^{r e d}(t)\right\| \leq h^{p} \sum_{\varphi \in S_{p}} \int_{\Delta_{p}(t)} N_{s_{1}}\left(Y_{\varphi(1)}\right) \cdots N_{s_{p}}\left(Y_{\varphi(p)}\right) d s_{1} \cdots d s_{p} \\
\leq h^{p} \prod_{j=1}^{p} \int_{0}^{t} N_{s}\left(Y_{j}\right) d s \leq h^{p} \prod_{j=1}^{p} g_{t}\left(Y_{j}\right)^{1 / 2} .
\end{gathered}
$$


Fix $X_{1}, \ldots, X_{m}$ and let $(\alpha, \beta)$ be a multi-index. Let $A_{\alpha \beta}$ be defined in the proof of Proposition 5.4 Note that, for all elements $f_{m n}$ in the basis $\mathcal{B}$, one has,

$$
\sup _{|s|<|t|} N_{s}\left(\left(f_{m n}, 0\right) \leq \varepsilon_{m n}(t), \quad \sup _{|s|<|t|} N_{s}\left(\left(0, f_{m n}\right) \leq \varepsilon_{m n}(t) .\right.\right.
$$

The above inequality shows that,

$$
\left\|A_{\alpha \beta} \operatorname{ad} O p_{h}^{\text {weyl }}\left(F_{X_{1}}\right) \otimes I \cdots \operatorname{ad} O p_{h}^{\text {weyl }}\left(F_{X_{m}}\right) \otimes I U_{h}^{r e d}(t)\right\| \leq \prod_{m n}\left(h|t| \varepsilon_{m n}(t)\right)^{\alpha_{m n}+\beta_{m n}} \prod_{j=1}^{m} \int_{0}^{t} h N_{s}\left(X_{j}\right) d s .
$$

Set

$$
V(t)=\left(\operatorname{ad} O p_{h}^{\text {weyl }}\left(F_{X_{1}}\right) \otimes I\right) \cdots\left(\operatorname{ad} O p_{h}^{\text {weyl }}\left(F_{X_{m}}\right) \otimes I\right) U_{h}^{r e d}(t) .
$$

The characterization theorem, Theorem 1.2 of [4, shows that $V(t)$ is associated with a function $V(t, q, p)$ by the Weyl calculus. In particular, it satisfies,

$$
|V(t, q, p)| \leq M(h, t) \prod_{j=1}^{m} \int_{0}^{t} h N_{s}\left(X_{j}\right) d s,
$$

where $M(h, t)$ is defined by (84). Using composition formulas that are valid if one of the operators is a linear form, one knows that,

$$
V(t, q, p)=h^{m} i^{-m} d^{m} U(t, q, p, h)\left(X_{1}, \ldots, X_{m}\right) .
$$

Consequently,

$$
\left|d^{m} U(t, q, p, h)\left(X_{1}, \ldots, X_{m}\right)\right| \leq M(h, t) \prod_{j=1}^{m} \int_{0}^{t} N_{s}\left(X_{j}\right) d s \leq M(h, t) \prod_{j=1}^{m} g_{t}\left(X_{j}\right)^{1 / 2} .
$$

The first inequality in (87) then follows.

Since $g_{t}(X)^{1 / 2} \leq K|t||X|$ then the second inequality in (87) is valid.

End of the Proof of Theorem 1.1 (iv). Extending the differential of order $m$ to a multilinear form on the complexified $H_{\mathbb{C}}^{2}$, the extension may be defined by,

$$
\mathcal{U}(t, X, h)=\sum_{j \geq 0} \frac{1}{j !} d^{j} U(t, 0,0, h)(X)^{j}, \quad X=(q, p) \in H_{\mathbb{C}}^{2}
$$

The estimate (3) comes from the fact that one may also write,

$$
\mathcal{U}(t, X+i Y, h)=\sum_{j \geq 0} \frac{1}{j !} d^{j} U(t, X, h)(i Y)^{j},
$$

for $X$ and $Y$ in $H^{2}$. 


\section{Proof of Theorem 1.1 (iii) (Semiclassical expansion of the re- duced propagator symbol).}

We study here the Weyl symbol $U(t, q, p, h)\left(t \in \mathbb{R},(p, q) \in H^{2}\right)$ of the operator $U_{h}^{\text {red }}(t)$ of (11) or (73). We aim to derive an asymptotic expansion of this symbol as $h$ goes to 0 ,

$$
U(t, q, p, h) \sim \sum_{j} g_{j}(t, q, p) h^{j}
$$

The functions $g_{j}(t, q, p)$ takes values in $\mathcal{L}\left(\left(\mathbb{C}^{2}\right)^{\otimes N}\right)$ similarly as the symbol itself. Since the symbol $U(t, q, p, h)$ satisfies (85)), it is natural that the functions $g_{j}$ satisfies,

$$
\frac{d}{d t} g_{0}(t, q, p)=-i H_{\text {int }}^{\text {free }}(t, q, p) g_{0}(t, q, p), \quad g_{0}(0, q, p)=I,
$$

where $H_{\text {int }}^{\text {free }}(t, q, p)$ is the matricial Weyl symbol of $H_{\text {int }}^{\text {free }}(t)$ defined in (68). For all $j \geq 1$, one should have,

$$
\frac{d}{d t} g_{j}(t, q, p)=-i H_{\text {int }}^{\text {free }}(t, q, p) g_{j}(t, q, p)-\frac{1}{2}\left\{H_{\text {int }}^{\text {free }}(t, \cdot), g_{j-1}(t, \cdot, h)\right\}(q, p), \quad g_{j}(0, q, p)=0 .
$$

Proposition 6.1. For all $(q, p)$ in $H^{2}$, the solutions $t \mapsto g_{j}(t, q, p)$ of the system (89) (90) (taking values in $\mathcal{L}\left(\left(\mathbb{C}^{2}\right)^{\otimes N}\right)$ ) are well defined on $\mathbb{R}$, and $g_{0}(t, q, p)$ is unitary. Moreover, one has for all multi-indices $(\alpha, \beta)$,

$$
\left|\partial_{q}^{\alpha} \partial_{p}^{\beta} g_{j}(t, q, p)\right| \leq \prod_{m n}\left(K|t| \varepsilon_{m n}(t)\right)^{\alpha_{m n}+\beta_{m n}},
$$

where $\varepsilon_{m n}(t)$ is defined in Proposition 5.1, for some positive real number $K$. The function $g_{j}(t, \cdot)$ then belongs to $S_{\infty}^{\text {mat }}(\mathcal{B}, K|t| \varepsilon(t))$.

Proof. The existence of time global solution and the unitarity of $g_{0}(t, q, p)$ are standard. Let us prove the bounds on $g_{0}$. Let $P_{1}, \ldots, P_{r}$ be a finite sequence of operators where each operator $P_{j}$ is either one

of the operators $\frac{\partial}{\partial q_{m n}}$ or $\frac{\partial}{\partial p_{m n}}$, the corresponding index $(m, n)$ being noted $\psi(j)$. Applying the operator $P_{1}$ on the two sides of (89) and solving the resulting system, we see that,

$$
P_{1} g_{0}(t, q, p)=-i \int_{0}^{t} g_{0}(t, q, p) g_{0}(s, q, p)^{\star}\left(P_{1} H_{\text {int }}^{\text {free }}(s, q, p)\right) g_{0}(s, q, p) d s .
$$

Iterating, one obtains,

$$
P_{1} \ldots P_{r} g_{0}(t, q, p)=(-i)^{r} \sum_{\varphi \in S_{r}} \int_{\Delta_{r}(t)} g_{0}(t, q, p) g_{0}\left(s_{r}, q, p\right)^{\star}\left(P_{\varphi(r)} H_{\text {int }}^{\text {free }}\left(s_{r}, q, p\right)\right)
$$




$$
\begin{gathered}
g_{0}\left(s_{r}, q, p\right) g_{0}\left(s_{r-1}, q, p\right)^{\star}\left(P_{\varphi(r-1)} H_{\text {int }}^{\text {free }}\left(s_{r-1}, q, p\right)\right) \\
g_{0}\left(s_{2}, q, p\right) g_{0}\left(s_{1}, q, p\right)^{\star}\left(P_{\varphi(1)} H_{\text {int }}^{\text {free }}\left(s_{1}, q, p\right)\right) g_{0}\left(s_{1}, q, p\right) d s_{1} \ldots d s_{r}
\end{gathered}
$$

where $S_{r}$ is the set of all bijections $\varphi$ of the set $\{1, \ldots, r\}$ and where $\Delta_{r}(t)$ is defined in (82), if $t>0$. Since $g_{0}(t, q, p)$ is unitary, one observes using (69),

$$
\left|P_{1} \ldots P_{r} g_{0}(t, q, p)\right| \leq \sum_{\varphi \in S_{r}} \prod_{j=1}^{r} \varepsilon_{\psi(\varphi(j))}(t) \int_{\Delta_{r}(t)} d s_{1} \cdots d s_{r} .
$$

The factors in front of the integral are all equals. The value of the integral is $|t|^{r} / r$ !. Consequently,

$$
\left|P_{1} \ldots P_{r} g_{0}(t, q, p)\right| \leq|t|^{r} \prod_{j=1}^{r} \varepsilon_{\psi(j)}(t) .
$$

Turning back to usual notations for multi-indices, one obtains (91) for $g_{0}$. Now suppose that the estimates (91) are valid for the integer $j-1$. Equations (89) (90) and Duhamel principle show that,

$$
g_{j}(t, q, p)=-\frac{1}{2} \int_{0}^{t} g_{0}(t, q, p) g_{0}(s, q, p)^{\star}\left\{H_{\text {int }}^{\text {free }}(t, \cdot), g_{j-1}(t, \cdot, h)\right\}(q, p) .
$$

The iteration hypothesis gives, if $|s|<|t|, g_{j}(s, \cdot)$ belongs to $S_{\infty}^{\text {mat }}(\mathcal{B},|t| \varepsilon(t))$. According to Proposition 3.2. the Poisson bracket $\left\{H_{\text {int }}^{\text {free }}(t, \cdot), U(t, \cdot, h)\right\}(q, p)$ belongs to a set $S_{\infty}^{\text {mat }}\left(\mathcal{B}, K^{\prime}|t| \varepsilon(t)\right)$. The function $g_{0}(t, \cdot)$ has already been seen to be in $S_{\infty}^{\text {mat }}(\mathcal{B},|t| \varepsilon(t))$. The estimates on $g_{j}$ then follows.

Proposition 6.2. The family of unitary matrices $g_{0}(t, q, p)$, solution to (89) and taking values in $\mathcal{L}\left(\left(\mathbb{C}^{2}\right)^{\otimes N}\right)$, satisfies, for all $s$ and $t$ in $\mathbb{R}$, for any $(q, p)$ in $H^{2}$,

$$
g_{0}(t, q, p) g_{0}(s, q, p)^{\star}=g_{0}\left(t-s, \chi_{s}(q, p)\right) .
$$

Proof. For any $s \in \mathbb{R}$, the two sides of the equality are solutions to the same differential system,

$$
\frac{d}{d t} F(t, q, p)=i H_{\text {int }}^{\text {free }}(t) F(t, q, p), \quad F(s, q, p)=I .
$$

For the right hand side, one uses the fact that $\left.H_{\text {int }}^{\text {free }}\left(t-s, \chi_{s}(q, p)\right)\right)=H_{\text {int }}^{\text {free }}(t, q, p)$. The Proposition then follows.

Proposition 6.3. Let $U(t, q, p, h)$ be the symbol of $U_{h}^{\text {red }}(t)$ and $g_{j}(t, q, p)(j \geq 1)$ be the solutions to (89) (90). Then we have for all integer $m$, 


$$
U(t, q, p, h)-\sum_{j=0}^{m} g_{j}(t, q, p) h^{j}=h^{m+1} R_{m}(t, q, p, h),
$$

where $R_{m}(t, \cdot, h)$ lies in a set $S_{\infty}^{\text {mat }}(\mathcal{B}, K|t| \varepsilon(t))$ and with $K$ being some constant.

Proof. Set,

$$
S_{m}(t, q, p, h)=\sum_{j=0}^{m} g_{j}(t, q, p) h^{j} .
$$

For $m=-1$, we agree that $S_{-1}(t, \cdot, h)=0$ and $R_{-1}(t, \cdot, h)=U(t, \cdot, h)$. The bounds on $R_{-1}$ are those of Theorem 1.1. Suppose that the Proposition is proved for the integer $m-1$. With notations (95) and (96), equation (85) reads as,

$$
\frac{d}{d t} U(t, \cdot, h)=-i H_{\text {int }}^{\text {free }}(t, \cdot) U(t, \cdot, h)-\frac{h}{2}\left\{H_{\text {int }}^{\text {free }}(t, \cdot), S_{m-1}(t, \cdot, h)+h^{m} R_{m-1}(t, \cdot, h)\right\} .
$$

Since the $g_{j}(t, q, p)$ satisfy (89) and (90), one has,

$$
\frac{d}{d t} S_{m}(t, \cdot, h)=-i H_{i n t}^{\text {free }}(t, \cdot) S_{m}(t, \cdot, h)-\frac{h}{2}\left\{H_{i n t}^{\text {free }}(t, \cdot), S_{m-1}(t, \cdot, h)\right\} .
$$

Since $g_{0}$ satisfies (94) and since $U-S_{m}$ is vanishing at $t=0$, Duhamel principle gives that,

$$
U(t, q, p, h)-S_{m}(t, q, p, h)=-\frac{h^{m+1}}{2} \int_{0}^{t} g_{0}(t, q, p) g_{0}(s, q, p)^{\star}\left\{H_{\text {int }}^{\text {free }}(s, \cdot), R_{m-1}(s, \cdot, h)\right\}(q, p) d s .
$$

According to iteration hypothesis, the function $R_{m-1}(s, \cdot, h)$ belongs to $S_{\infty}^{\text {mat }}(\mathcal{B},|t| \varepsilon(t))$. In view of Proposition 3.2, the Poisson bracket $\left\{H_{\text {int }}^{\text {free }}(t, \cdot), R_{m-1}(s, \cdot, h)\right\}(q, p)$ belongs to a set $S_{\infty}^{\text {mat }}\left(\mathcal{B}, K^{\prime}|t| \varepsilon(t)\right)$. According to Proposition 6.1, the function $g_{0}(t, \cdot)$ est dans $S_{\infty}^{m a t}(\mathcal{B},|t| \varepsilon(t))$. The proof of the Proposition is completed

\section{Proof of Theorem 1.2. (Consequences of analyticity proper- ties.)}

Theorem 7.1. Let $F$ be a function in the set $S_{\infty}^{\text {mat }}(\mathcal{B}, \varepsilon)$. Suppose that, for all integers $m$, for any vectors $X_{1}, . . X_{m}$ in $H^{2}$,

$$
\left|d^{m} F(q, p)\left(X_{1}, . . X_{m}\right)\right| \leq M \prod_{j=1}^{m}\left(K\left|X_{j}\right|\right) .
$$


Then, we have for all $X$ and $Y$ in $H^{2}$, for any a and $b$ in $\left(\mathbb{C}^{2}\right)^{\otimes N}$ with norm 1 ,

$$
\left|<O p_{h}^{\text {weyl }}(F)\left(\Psi_{X, h} \otimes a\right),\left(\Psi_{Y, h} \otimes b\right)>\right| \leq M e^{K|X-Y|-\frac{1}{4 h}|X-Y|^{2}} .
$$

Proof. Under our hypotheses, the function $F$ has a stochastic extension $\widetilde{F}$ in $L^{1}\left(B^{2}, \mu_{B^{2}, t}\right)$ (see Section 2.2), for all $t>0$. For any $t>0$, the operator,

$$
\left(H_{t} F\right)(X)=\int_{B^{2}} \widetilde{F}(X+Y) d \mu_{B^{2}, t}(Y)
$$

is considered as the heat operator. The function $H_{h / 2} F(\cdot)$ also satisfies,

$$
\left|d^{m} H_{h / 2} F(q, p)\left(X_{1}, \ldots, X_{m}\right)\right| \leq M \prod_{j=1}^{m}\left(K\left|X_{j}\right|\right) .
$$

Consequently, as in the proof of Theorem 1.1 (end of Section [5), functions $F$ and $H_{h / 2} F$ are extended to holomorphic functions on $H_{\mathbb{C}}^{2}$, taking values in $\mathcal{L}\left(\left(\mathbb{C}^{2}\right)^{\otimes N}\right)$, denoted $\mathcal{F}$ and $H_{h / 2} \mathcal{F}(\cdot, h)$ and satisfying,

$$
\left|H_{h / 2} \mathcal{F}(X+i Y, h)\right| \leq M e^{K|Y|}, \quad X+i Y \in H_{\mathbb{C}}^{2} .
$$

The bi-symbol of $O p_{h}^{\text {weyl }}(F)$ defined in (24) is,

$$
S_{h}\left(O p_{h}^{\text {weyl }}(F)\right)\left(q, p, q^{\prime}, p^{\prime}\right)=\left(H_{h / 2} \mathcal{F}\right)\left(\frac{q+i p}{2}+\frac{q^{\prime}-i p^{\prime}}{2}, \frac{p-i q}{2}+\frac{p^{\prime}+i q^{\prime}}{2}, h\right) .
$$

Indeed, one knows that the bi-symbol of an operator is a function on $H^{2} \times H^{2}$ which is holomorphic with respect to the first variable and anti-holomorphic with respect to the seconde variable $H^{2}$ identified with $H_{\mathbb{C}}$. The above function is the only one sharing theses properties and whose restriction to the diagonal equals to $H_{h / 2} F(\cdot)$, which is the Wick symbol of the operator $O p_{h}^{\text {weyl }}(F)$ (from [3], Theorem 7.1). For all $X$ and $Y$ in $H^{2}$, for any $a$ and $b$ in $E$ with unit norms, one deduces from (24),

$$
<O p_{h}^{\text {weyl }}(F)\left(\Psi_{X, h} \otimes a\right),\left(\Psi_{Y, h} \otimes b\right)>=S_{h}\left(O p_{h}^{\text {weyl }}(F)\right)(X, Y)(a) \cdot b<\Psi_{X, h}, \Psi_{Y, h}>,
$$

thus, setting $X=(q, p), Y=\left(q^{\prime}, p^{\prime}\right)$,

$$
=\left(H_{h / 2} \mathcal{F}\right)\left(\frac{q+i p}{2}+\frac{q^{\prime}-i p^{\prime}}{2}, \frac{p-i q}{2}+\frac{p^{\prime}+i q^{\prime}}{2}, h\right)(a) \cdot b \quad\left\langle\Psi_{X, h}, \Psi_{Y, h}>.\right.
$$

From (100) and using a standard equality concerning the scalar product of coherent states ([4], formula (2.3)), we have indeed (98). 
Proof of Theorem 1.2. One has,

$$
\begin{gathered}
<e^{-i \frac{t}{h} H(h)}\left(\Psi_{X, h} \otimes a\right),\left(\Psi_{Z, h} \otimes b\right)>=<e^{-i \frac{t}{h} H_{0}} U_{h}^{r e d}(t)\left(\Psi_{X, h} \otimes a\right),\left(\Psi_{Z, h} \otimes b\right)> \\
=<U_{h}^{r e d}(t)\left(\Psi_{X, h} \otimes a\right),\left(\Psi_{\chi_{-t}(Z), h} \otimes b\right)>.
\end{gathered}
$$

It is then sufficient to apply Theorem 7.1 with $F(q, p)=U(t, q, p, h)$.

\section{References}

[1] A. Abdesselam, The ground state energy of the massless spin-boson model, Ann. Henri Poincaré 12 (2011), no. 7, 1321-1347.

[2] L. Amour, L. Jager, J. Nourrigat, On bounded pseudodifferential operators in a high-dimensional setting, preprint, Proc. Amer. Math. Soc. 143 (2015), no. 5, 2057-2068.

[3] L. Amour, L. Jager, J. Nourrigat, On bounded pseudodifferential operators in Wiener spaces, J. Funct. Anal. 269 (2015), no. 9, 2747-2812.

[4] L. Amour, R. Lascar, J. Nourrigat, Beals characterization of pseudodifferential operators in Wiener spaces, Appl. Math. Res. Express (2016).

[5] L. Amour, J. Nourrigat, Hamiltonian systems and semiclassical dynamics for interacting spins in QED, arXiv:1512.08429

[6] A. Arai, M. Hirokawa, On the existence and uniqueness of ground states of a generalized spin-boson model, J. Funct. Anal. 151 (1997), no. 2, 455-503.

[7] V. Bach, J. Fröhlich, I. M. Sigal, Quantum electrodynamics of confined nonrelativistic particles, Adv. Math. 137 (1998), no. 2, 299-395.

[8] F. Bloch, Nuclear Induction, Physical Review 70 (1946), 460-473.

[9] L. Boutet de Monvel, P. Kree, Pseudodifferential operators and Gevrey classes, Ann. Institut Fourier 17, (1967), 295-323.

[10] Claude Cohen-Tannoudji, Jacques Dupont-Roc et Gilbert Grynberg, Photons et atomes. Introduction à l'electrodynamique quantique, EDP Sciences CNRS Editions, 2001. 
[11] Claude Cohen-Tannoudji, Jacques Dupont-Roc et Gilbert Grynberg, Processus d'interaction entre photons et atomes, EDP Sciences CNRS ditions, 2001.

[12] M. Combescure, D. Robert, Coherent states and applications in mathematical physics, Theoretical and Mathematical Physics, Springer, Dordrecht, 2012.

[13] J. Dereziński, C. Gérard, Asymptotic completeness in quantum field theory. Massive Pauli-Fierz Hamiltonians, Rev. Math. Phys. 11 (1999), no. 4, 383-450.

[14] W. De Roeck, M. Griesemer, A. Kupiainen, Asymptotic completeness for the massless spin-boson model, Adv. Math. 268 (2015), 62-84.

[15] M. Dimassi, J. Sjöstrand, Spectral asymptotics in the semi-classical limit, London Mathematical Society Lecture Note Series, 268, Cambridge University Press, Cambridge, 1999.

[16] G. B. Folland, Harmonic analysis of the de Rham complex on the sphere, J. Reine Angew. Math. 398 (1989), 130-143.

[17] J. Fröhlich, On the infrared problem in a model of scalar electrons and massless, scalar bosons, Ann. Inst. H. Poincaré Sect. A (N.S.) 19 (1973) 1-103.

[18] J. Fröhlich, On the infrared problem in a model of scalar electrons and massless scalar bosons, Fortschr. Phys. 22 (1974) 159-198.

[19] W. Greiner, J. Reinhardt, Field Quantization, Springer-Verlag, Berlin Heidelberg (1996).

[20] L. Gross, Measurable functions on Hilbert space, Trans. Amer. Math. Soc. 105 (1962) 372-390.

[21] L. Gross, Abstract Wiener spaces, Proc. 5th Berkeley Sym. Math. Stat. Prob, 2, (1965), 31-42.

[22] L. Gross, Abstract Wiener measure and infinite dimensional potential theory, in Lectures in modern Analysis and applications, II, Lecture Notes in Math 140, 84-116, Springer (1970).

[23] B. Helffer, Théorie spectrale pour des opérateurs globalement elliptiques, Astérisque, 112, Société Mathématique de France, Paris, 1984.

[24] M. Hübner, H. Spohn, Spectral properties of the spin-boson Hamiltonian, Annales de l'I. H. P., Section A, tome 62, 3 (1995), p. 289-323.

[25] M. Hübner, H. Spohn, Radiative decay: nonperturbative approaches, Rev. Math. Phys. 7 (1995), no. $3,363-387$. 
[26] A. Ikeda, Y. Tanigushi, Spectra and eigenforms of the Laplacien on $S^{n}$ and $P^{n}(\mathbb{C})$, Osaka J. Math, 15 (1978), 515-546.

[27] S. Janson, Gaussian Hilbert spaces, Cambridge Tracts in Math. 129, Cambridge Univ. Press (1997).

[28] H. H. Kuo, Gaussian measures in Banach spaces. Lecture Notes in Mathematics, Vol. 463. Springer, Berlin-New York, 1975.

[29] B. Lascar, Une classe d'opérateurs elliptiques du second ordre sur un espace de Hilbert, J. Funct. Anal. 35 (1980), no. 3, 316-343.

[30] E. Lieb, M. Loss, A note on polarization vectors in quantum electrodynamics. Comm. Math. Phys. 252 (2004), no. 1-3, 477-483.

[31] A. Martinez, An introduction to semiclassical and microlocal analysis, Universitext, Springer-Verlag, New York, 2002.

[32] E. Peskin, D Schroeder, Quantum field theory, Westview Press, 1995.

[33] M. Reed, B. Simon, Methods of modern mathematical physics, Vol I, Functional Analysis, Academic Press, 1975.

[34] M. Reed, B. Simon, Methods of modern mathematical physics, Vol II, Fourier Analysis, selfadjointness, Academic Press, 1975.

[35] F.A. Reuse, Electrodynamique et Optique Quantiques, Presses Polytechniques et Universitaires Romandes, Lausanne, 2007.

[36] D. Robert, Autour de l'approximation semi-classique, Progress in Mathematics, 68, Birkhäuser Boston, Inc., Boston, MA, 1987

[37] B. Simon, The $P(\Phi)_{2}$ Euclidean (Quantum) Field theory, Princeton Series in Physics, Princeton Univ. Press, Princeton (1974).

[38] H. Spohn, Dynamics of charged particles and their radiation field, Cambridge University Press, Cambridge, 2004.

[39] A. Unterberger, Les opérateurs métadifférentiels, in Complex analysis, microlocal calculus and relativistic quantum theory, Lecture Notes in Physics 126 (1980) 205-241.

[40] S. Weinberg, The quantum theory of fields, Cambridge University Press, 1996. 
[41] M. Zworski, Semiclassical analysis, Graduate Studies in Mathematics, 138. American Mathematical Society, Providence, RI, 2012.

laurent.amour@univ-reims.fr

LMR EA 4535 and FR CNRS 3399, Université de Reims Champagne-Ardenne, Moulin de la Housse, BP 1039, 51687 REIMS Cedex 2, France.

rlascar@math.univ-paris-diderot.fr

Institut Mathématique de Jussieu UMR CNRS 7586, Analyse Algébrique, 4 Place Jussieu, 75005 Paris, France.

jean.nourrigat@univ-reims.fr

LMR EA 4535 and FR CNRS 3399, Université de Reims Champagne-Ardenne, Moulin de la Housse, BP 1039, 51687 REIMS Cedex 2, France. 